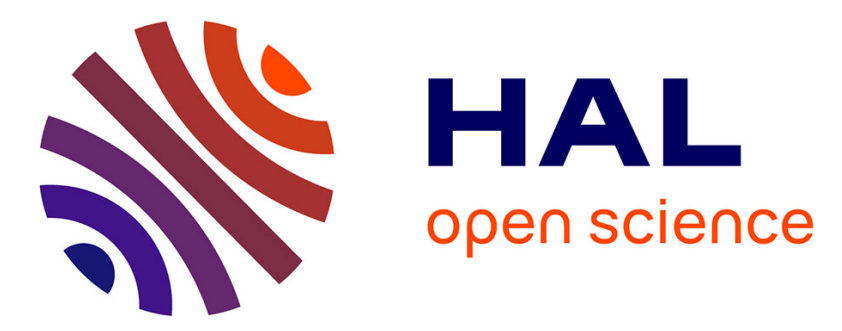

\title{
Precise test of quantum electrodynamics and determination of fundamental constants with $\mathrm{HD}+$ ions
}

S. Alighanbari, G. S. Giri, Florin Lucian Constantin, V. I. Korobov, S. Schiller

\section{To cite this version:}

S. Alighanbari, G. S. Giri, Florin Lucian Constantin, V. I. Korobov, S. Schiller. Precise test of quantum electrodynamics and determination of fundamental constants with HD+ ions. Nature, 2020, 581 (7807), pp.152-158. 10.1038/s41586-020-2261-5 . hal-03058791

\section{HAL Id: hal-03058791 \\ https://hal.science/hal-03058791}

Submitted on 11 Dec 2020

HAL is a multi-disciplinary open access archive for the deposit and dissemination of scientific research documents, whether they are published or not. The documents may come from teaching and research institutions in France or abroad, or from public or private research centers.
L'archive ouverte pluridisciplinaire HAL, est destinée au dépôt et à la diffusion de documents scientifiques de niveau recherche, publiés ou non, émanant des établissements d'enseignement et de recherche français ou étrangers, des laboratoires publics ou privés.

$$
\text { Copyright }
$$




\title{
Precise test of quantum electrodynamics and determination of
}

\section{fundamental constants with $\mathrm{HD}^{+}$ions}

\author{
S. Alighanbari ${ }^{1}$, G. S. Giri ${ }^{1}$, F. L. Constantin ${ }^{1,2}$, V. I. Korobov ${ }^{3} \&$ S. Schiller ${ }^{1, \dagger}$ \\ ${ }^{1}$ Institut für Experimentalphysik, Heinrich-Heine-Universität Düsseldorf, Düsseldorf, Germany. \\ ${ }^{2}$ Laboratoire PhLAM CNRS UMR 8523, Université Lille 1, Villeneuve d’Ascq, France. \\ ${ }^{3}$ Bogoliubov Laboratory of Theoretical Physics, Joint Institute for Nuclear Research, Dubna, Russia. \\ †step.schiller@hhu.de
}

Bound three-body quantum systems are important systems for fundamental physics ${ }^{1,2}$. They enable tests of quantum electrodynamics theory and provide access to the fundamental constants of atomic physics and to nuclear properties. Molecular hydrogen ions, the simplest molecules, are representatives of this class ${ }^{3}$. The metastability of the vibration-rotation levels in their ground electronic states offers the potential for extremely high spectroscopic resolution. Consequently, these systems provide independent access to the Rydberg constant $\left(R_{\infty}\right)$, the ratios of electron mass to proton mass $\left(m_{\mathrm{e}} / m_{\mathrm{p}}\right)$ and electron mass to deuteron mass $\left(m_{\mathrm{e}} / m_{\mathrm{d}}\right)$, proton and deuteron nuclear radii, and high-level tests of quantum electrodynamics ${ }^{4}$. Conventional spectroscopy techniques for molecular ions ${ }^{5-14}$ have long been unable to provide precision competitive with that of ab initio theory, which has strongly improved in recent years ${ }^{15}$. Here we improve our rotational spectroscopy technique for a sympathetically cooled cluster of molecular ions stored in a linear radiofrequency trap ${ }^{16}$ by nearly two orders in accuracy. We measured a set of hyperfine components of the rotational transition. An evaluation resulted in the most accurate test of a quantum-three-body prediction so far, at the level of $5 \times 10^{-11}$, limited by the current uncertainties of the fundamental constants. We determined the value of the fundamental constants $R_{\infty} m_{\mathrm{e}}\left(m_{\mathrm{p}}^{-1}+m_{\mathrm{d}}^{-1}\right)$ and $\boldsymbol{m}_{\mathrm{e}} / \boldsymbol{m}_{\mathrm{p}}$ with a fractional uncertainty of $\mathbf{2} \times 10^{-11}$, in agreement with but more precise than current CODATA2018 values. These results are also strong evidence of the correctness of previous key high-precision measurements. Furthermore, our work yields a more than 20 -fold stronger bound for a hypothetical fifth force between a proton and a deuteron. 
Since the inception of quantum mechanics, the precise understanding of three-body systems has represented a challenging fundamental physics problem. Its detailed study, both theoretical and experimental, is an ongoing effort, with a strong rate of improvement. Different three-body systems (for example, the helium atom, lithium ion, helium-like ions, antiprotonic helium atom and molecular hydrogen ions (MHIs)) provide the opportunity to test our understanding of quantum physics at the highest levels, in particular, the theory of quantum electrodynamics (QED). In doing so, important fundamental constants of physics (such as the Rydberg constant $R_{\infty}$, fine-structure constant $\alpha$, electron mass $m_{\mathrm{e}}$, proton mass $m_{\mathrm{p}}$, deuteron mass $m_{\mathrm{d}}$ and antiproton mass) and particular nuclear properties, such as charge radii, electric quadrupole moments and charge-current moments, can be determined.

The MHIs ( $\mathrm{HD}^{+}, \mathrm{H}_{2}^{+}$and so on) are molecular three-body systems containing two heavy particles and one light particle (electron). The electronic ground state supports hundreds of metastable rotation-vibration levels. A small subset of them have been studied with different experimental techniques and concerning different aspects since the mid-1960s ${ }^{5-14,17}$ (for an early review, see ref. ${ }^{3}$ ). Over the past decade, the MHIs have come into focus because of their relevance for the metrology of the particle masses ${ }^{4,18-21}$. These can be determined from rotationvibration spectroscopic data, an approach independent of the established technique of mass spectrometry in ion traps. An additional opportunity is the determination of the Rydberg constant $R_{\infty}$ and the proton charge radius, independently from the established technique of atomic hydrogen spectroscopy ${ }^{22-24}$. The precise value of these constants has been called into question in recent years in connection with the "proton radius puzzle" ${ }^{25}$, and therefore alternative and independent approaches for its determination are highly desirable.

The ab initio theory of the MHIs has made enormous progress in precision over the past 20 years $^{26-28}$, reducing the uncertainty by four orders of magnitude. It currently stands at $1.4 \times 10^{-11}$ fractionally for the fundamental rotational transition frequency and $7 \times 10^{-12}$ for vibrational transitions, ignoring spin-structure effects ${ }^{15}$. These uncertainties are smaller than the current (CODATA $2018^{29}$ ) uncertainties of the masses $m_{\mathrm{e}}, m_{\mathrm{p}}$ and $m_{\mathrm{d}}$, pointing at the potential of MHI spectroscopy for the metrology of fundamental constants. Here we perform precision spectroscopy of the fundamental rotational transition of $\mathrm{HD}^{+}$. Fundamental constants can be derived by comparison of the measured transition frequency $f^{(\exp )}$ with the prediction 
$f^{(\text {theor })}=2 c R_{\infty}\left(m_{\mathrm{e}} / \mu_{\mathrm{pd}}\right) F_{\text {spin-avg }}^{(\text {theor })}$, where $\mu_{\mathrm{pd}}=m_{\mathrm{p}} m_{\mathrm{d}} /\left(m_{\mathrm{p}}+m_{\mathrm{d}}\right)$ is the reduced nuclear mass, $\mathrm{c}$ is the speed of light, and $F_{\text {spin-avg }}^{(\text {theor })} \approx 0.244591781951(33)_{\text {theory }}(11)_{\text {CODATA2018 }}$ is a dimensionless normalized frequency computed ab initio, neglecting the hyperfine interactions. $F_{\text {spin-avg }}^{(\text {theor })}$ encompasses, besides the dominant non-relativistic (Schrödinger) part, essential relativistic, nuclear-size-related and radiative contributions. The nuclear charge radius values $\left(r_{\mathrm{p}}, r_{\mathrm{d}}\right)$ are from the CODATA2018 adjustment that took into account the muonic hydrogen spectroscopy results. Whereas the uncertainty of $F_{\text {spin-avg }}^{\text {(theor) }}$ due to theory is $1.4 \times 10^{-11}$, the uncertainty originating from the CODATA 2018 uncertainties of the fundamental constants is smaller, $4.4 \times 10^{-12}$, and stems from the uncertainties of $r_{\mathrm{p}}$ and $r_{\mathrm{d}}$.

Apart from a matching comparison with a 50-year-old radiofrequency (RF) spectroscopy benchmark result on $\mathrm{H}_{2}^{+}$(ref. ${ }^{17}$ ), the ab initio theory could not be tested experimentally at a competitive level, owing to lack of suitable experimental methods. Apart from a few exceptions, the spectroscopic resolution in rotational and vibrational molecular ion spectroscopy in general has been limited by Doppler broadening. Although this broadening can be minimized by trapping molecular ions in an RF trap and sympathetically cooling them by atomic ions, their effective temperature remains of the order of $10 \mathrm{mK}$, leading to Doppler-limited linewidths not lower than $5 \times 10^{-8}$ fractionally $^{12}$. Unresolved hyperfine structure increases linewidths again ${ }^{11,14}$, posing a roadblock for testing theory at more precise levels.

Only recently, new methods have been introduced that open up the next generation of precision experiments $^{30,31}$. Specifically for rotational spectroscopy, we have shown ${ }^{16}$ that subDoppler spectroscopy is possible for a radiation propagation direction transverse to the 'long' axis of the molecular ion cluster (trapped ion cluster transverse excitation spectroscopy, TICTES). The small motional amplitude of the ions along the spectroscopy wave propagation direction compared with its wavelength allows reaching the Lamb-Dicke regime. In the first demonstration $^{16}$, a fractional line resolution of $1 \times 10^{-9}$ (full-width at half-maximum (FWHM) relative to absolute frequency) was obtained. 
Here we improve the resolution of TICTES by more than two orders of magnitude. This enables a detailed direct study of the fundamental rotational transition of $\mathrm{HD}^{+}$, whose hyperfine spectrum and Zeeman splittings are resolved and systematic effects are determined.

Comparison with our improved theory and new analysis method allows us to establish agreement between theory and experiment at the $5 \times 10^{-11}$ level (limited by CODATA2018 uncertainties), not only representing the most accurate test of a molecular three-body system so far, but also demonstrating the power of TICTES, a method applicable to a plethora of molecular ions.

\section{The experiment}

We performed spectroscopy of the fundamental rotational transition $(v, N)=(0,0) \rightarrow\left(v^{\prime}\right.$, $\left.N^{\prime}\right)=(0,1)$ at $1.3 \mathrm{THz} . v$ and $N$ are the vibrational and rotational quantum numbers, respectively. See Extended Data Fig. 1 for the experimental scheme. The fractional population of $\mathrm{HD}^{+}$ions in the lower spectroscopy state $(0,0)$ is enhanced using rotational laser cooling ${ }^{32}$. The transition is detected by resonance-enhanced multiphoton dissociation (REMPD) ${ }^{33}$. See Extended Data Fig. 2 for typical data. To achieve a spectroscopy wave with narrow linewidth, high frequency stability and high accuracy, a GPS-monitored, hydrogen-maser-referenced terahertz frequency multiplier is used ${ }^{16,34}$. Compared with our previous work $^{16}$, we performed measurements for different magnetic-, electric- and light-field strengths, and minimized the terahertz wave power. These extensive measurements were enabled by improvements in the long-term stability of the apparatus and improved detection schemes.

The $\mathrm{HD}^{+}$molecule has spin structure in both the lower and the upper rotational levels, due to the presence of (1) the intrinsic spins of the electron $\left(\mathbf{s}_{\mathrm{e}}\right)$, proton $\left(\mathbf{I}_{\mathrm{p}}\right)$ and deuteron $\left(\mathbf{I}_{\mathrm{d}}\right)$, and (2) of the rotational angular momentum $\mathbf{N}$ (Fig. 1). For state description, we use the angular momentum coupling scheme $\mathbf{G}_{1}=\mathbf{s}_{\mathrm{e}}+\mathbf{I}_{\mathrm{p}}, \mathbf{G}_{2}=\mathbf{G}_{1}+\mathbf{I}_{\mathrm{d}}, \mathbf{F}=\mathbf{G}_{2}+\mathbf{N}$ (ref. ${ }^{35}$ ), where $\mathbf{F}$ is the total angular momentum. The rotational transition encompasses 32 hyperfine components $f_{i}$ in absence of a magnetic field; of these, ten are favoured (strong) (Fig. 1). Their frequencies $f_{12}, \ldots$, $f_{21}$ lie within a range of $45 \mathrm{MHz}$ around $f_{\text {spin-avg }} \approx 1.314 \mathrm{THz}$. Averaging over these ten

components with appropriate weights yields the 'spin-averaged' frequency $f_{\text {spin-avg }}{ }^{36}$. Here we measured six hyperfine components, $f_{12}, f_{14}, f_{16}, f_{19}, f_{20}$ and $f_{21}$. 
Figure 2 shows the measured transitions, in the presence of a small magnetic field. The different linewidths are due to the different terahertz wave intensities used and due to the different transition dipole moments. Line 19 includes the two transitions between states of maximum total angular momentum $F$ and maximum projection quantum number $m_{\mathrm{F}},(F=2$, $\left.m_{\mathrm{F}}= \pm 2\right) \rightarrow\left(F^{\prime}=3, m_{\mathrm{F}}^{\prime}= \pm 3\right)$, denoted by $19_{ \pm}$, whose Zeeman shift is purely linear, according to theory ${ }^{37}$. The two components were observed at lower resolution and with unresolved Zeeman splitting in ref. ${ }^{16}$. One Zeeman component (19-) measured at particularly low intensity exhibited a full linewidth of $4 \mathrm{~Hz}$, or $3 \times 10^{-12}$ fractionally, indicating the potential of the experimental technique in the context of mass determination. For line 16, we measured a Zeeman pair $m_{\mathrm{F}}= \pm 1 \rightarrow m_{\mathrm{F}}^{\prime}= \pm 2$ (denoted by $16_{ \pm}$), split by a linear Zeeman shift and weakly shifted by a common quadratic Zeeman shift, and a component $16_{0}: m_{\mathrm{F}}=0 \rightarrow m_{\mathrm{F}}^{\prime}=0$, which exhibits a moderate quadratic Zeeman $\operatorname{shift}^{37}$. For the remaining lines, we measured only the $m_{\mathrm{F}}=0$ $\rightarrow m_{\mathrm{F}}^{\prime}=0$ Zeeman components.

\section{Systematic shifts}

For an accurate comparison between theoretical transition frequencies (computed assuming an absence of perturbing fields) and experimental values (measured in presence of such fields), the systematic shifts must be taken into account. We determined them experimentally. The dominant systematic effect is the Zeeman shift. For a nominal RF drive amplitude, we measured the frequency shifts of all considered components as a function of applied magnetic field. The shifts are consistent with the theoretically calculated ones, except for small deviations. We obtained the transition frequencies corresponding to zero magnetic field by extrapolation.

The quadratic Stark shift due to the ion trap's electric field $E(t)$, oscillating at comparatively low (RF) frequency and leading to a mean-square value $\left\langle E(t)^{2}\right\rangle$, is a second shift, of lower magnitude. For a nominal magnetic field, we measured the frequency shifts of all considered components for a set of trap RF amplitudes. All shifts were found to increase with amplitude, with values in the range of 0.5 to $1.2 \mathrm{kHz} \mathrm{kV}^{-2}$. We determined the frequencies corresponding to zero RF-field amplitude by extrapolation. For additional information, see Methods and Extended Data Fig. 3. 
Table 1 presents the experimental transition frequencies $f_{i}^{(\exp )}$ (corrected for the systematic shifts) and their uncertainties. The uncertainties result from the number of frequency measurements, which were taken at different RF drive settings and different magnetic-field settings, and the statistical uncertainties of the frequency measurements. The lowest experimental uncertainty is achieved for line $16, u\left(f_{16}^{(\exp )}\right)=0.017 \mathrm{kHz}$ (fractional uncertainty $u_{\mathrm{r}}$ $\left.=1.3 \times 10^{-11}\right)$. This represents the best performance level of the TICTES technique as currently implemented.

\section{Theory}

For a compelling comparison between theory and the experimental data, highly precise theoretical predictions and qualified estimates of their uncertainties are essential. The ab initio transition frequency $f_{i}^{\text {(theor) }}$ of each hyperfine component $i$ is the sum of two contributions, $f_{\text {spin-avg }}^{\text {(theor }}+f_{\text {spin, } i}^{\text {(ther) }}$. The dominant contribution is

$$
f_{\text {spin-avg }}^{(\text {theor })}=1,314,925,752.896(18)_{\text {theory }}(61)_{\text {CODATA2018 }} \mathrm{kHz}
$$

computed $^{15}$ including all relativistic and radiative corrections up to the relative order $\alpha^{5}$ and partially including contributions of the order $\alpha^{6}$ (Table 2). The value $f_{\text {spin-avg }}^{\text {(theor }}$ is updated from the value reported in ref. ${ }^{16}$ by using CODATA $2018^{29}$ updates of the Rydberg constant, the particle masses (in atomic mass units, $u$ ), the proton charge radius and the deuteron charge radius. The theory uncertainty is estimated as $\mathrm{u}\left(f_{\text {spin-avg }}^{\text {(theor) }}\right) \approx 0.018 \mathrm{kHz}$, while the larger CODATA 2018 uncertainty, $\mathrm{u}_{\text {CODATA2018 }}\left(f_{\text {spin-avg }}^{\text {(theor) }}\right) \approx 0.061 \mathrm{kHz}$, is dominated by the uncertainties of the particle masses.

A spin frequency contribution $f_{\text {spin }, i}^{\text {(theor }}$ is the difference of the spin structure energies of the upper and lower spin states involved in the transition. For the favoured transitions measured here, the $f_{\mathrm{spin}, i}^{(\text {theor })}$ are of the order of $10 \mathrm{MHz}$. The spin contributions are computed by diagonalizing the Breit-Pauli spin Hamiltonian of ref. ${ }^{35}$. The various terms of this Hamiltonian are proportional to coefficients $E_{k}, E_{k}^{\prime}$, computed ab initio (Extended Data Table 1). The spin Hamiltonian of the $N=0$ level necessitates two coefficients, $E_{4}$ and $E_{5}$, while the $N=1$ level necessitates nine, $E_{1}^{\prime}, \ldots, E_{9}^{\prime}$. 
The coefficients $E_{4}, E_{4}^{\prime}$ and $E_{5}, E_{5}^{\prime}$ describe the dominant $\mathbf{s}_{\mathbf{e}} \cdot \mathbf{I}_{\mathbf{p}}$ and $\mathbf{s}_{\mathbf{e}} \cdot \mathbf{I}_{\mathbf{d}}$ interaction, respectively, and have been calculated with high theoretical precision, including all corrections of the order $\alpha^{2} E_{\mathrm{F}} / h$ and the leading corrections of the order $\alpha^{3} E_{\mathrm{F}} / h$, where $E_{\mathrm{F}} \approx h(1.4 \mathrm{GHz})$ is the Fermi contact energy for the hyperfine splitting in atomic hydrogen and $h$ is Planck's constant ${ }^{38}$. The fractional theoretical uncertainties of these spin Hamiltonian coefficients are of the order $\alpha^{3}$; they are estimated as $\varepsilon_{\mathrm{F}}=1 \times 10^{-6}$. Furthermore, the theory errors are expected to be nearly equal: $\Delta E_{4}{ }^{\text {(theor) }} \approx \Delta E_{4}^{\prime}{ }^{\text {(theor) }}$ and $\Delta E_{5}{ }^{\text {(theor) }} \approx \Delta E_{5}^{\prime}{ }^{\text {(theor) }}$ (Methods).

The other spin coefficients, $E_{1}^{\prime}, E_{2}^{\prime}, E_{3}^{\prime}, E_{6}^{\prime}, E_{7}^{\prime}, E_{8}^{\prime}$, and $E_{9}^{\prime}$, have been obtained within the Breit-Pauli approximation. We computed them using our most precise non-relativistic nonadiabatic molecular variational wave functions (Methods, Extended Data Table 1). The omitted terms are of the relative order $\alpha^{2}$. References ${ }^{38,39}$ lead us to estimate a common fractional theory uncertainty equal to $\alpha^{2}=\varepsilon_{0} \approx 5 \times 10^{-5}$.

To determine the impact of the theory uncertainty of a particular Hamiltonian coefficient on a particular spin frequency, we consider the quantity $\gamma_{i, k}^{\prime} \Delta E_{k}^{\prime(\text { theor })}, \gamma_{i, k}^{\prime}$ is the derivative $\gamma_{i, k}^{\prime}=\partial E_{\mathrm{spin}, i}^{\prime}\left(E_{1}^{\prime}, \ldots, E_{9}^{\prime}\right) / \partial E_{k}^{\prime}$, and similarly for the coefficients relevant to the lower spin level. The $\gamma$ values are reported in Extended Data Table 1. Assuming equal theory errors for the pairs $\left(E_{4}, E_{4}^{\prime}\right)$ and $\left(E_{5}, E_{5}^{\prime}\right)$, we conservatively estimate the total theory uncertainty of the spinfrequency contribution with the following expression

$$
u\left(f_{\text {spin }, i}^{(\text {theor })}\right)=\varepsilon_{\mathrm{F}} \sum_{4,5}\left|\gamma_{i, k}^{\prime} E_{k}^{\prime}-\gamma_{i, k} E_{k}\right|+\varepsilon_{0} \sum_{1,2,3,6,7,8,9}\left|\gamma_{i, k}^{\prime} E_{k}^{\prime}\right|
$$

The minus sign in the first sum embodies the assumption of equal errors and correlation, $\Delta E_{4}{ }^{\text {(theor) }}=\Delta E_{4}^{\prime}{ }^{\text {(theor) }} \approx \varepsilon_{\mathrm{F}}$ and $\Delta E_{5}{ }^{\text {(theor) }}=\Delta E_{5}^{\prime}{ }^{\text {(theor) }} \approx \varepsilon_{\mathrm{F}}$. The similarity $\gamma_{4} \approx \gamma_{4}^{\prime}$ and $\gamma_{5} \approx \gamma_{5}^{\prime}$ for the lower and upper rotational levels then leads to a strong suppression of the contributions related to the theory errors of $E_{4}, E_{4}^{\prime}, E_{5}$ and $E_{5}^{\prime}$. This results in the spin-frequency uncertainties shown in Table 1 (column 6). They dominate the total uncertainty of the transition frequencies $f_{i}^{\text {(theor) }}$.

\section{Comparison between theory and experiment}

Table 1 presents the comparison between the theory and experimental data of the individual hyperfine components of the rotational transition. We find agreement for all lines, within the combined uncertainties of theory and experiment. The agreement is most stringent for line 16, 
and it is limited by the prediction's total uncertainty $u\left(f_{16}^{\text {(theory })}\right) \approx 0.21 \mathrm{kHz}$, or about $1.5 \times 10^{-10}$ fractionally. The agreement is far less stringent than the roughly ten times lower experimental uncertainty would allow. The precise experimental value can therefore serve as a benchmark for tests of future improved spin-structure calculations.

Frequencies related to only the spin structure of the molecule can be obtained from rotational frequency differences $\Delta f_{i, j}=f_{i}-f_{j}=f_{\text {spin }, i}-f_{\text {spin }, j}$, where the spin-averaged frequency is cancelled. All deviations between experiment and theory are smaller than $0.42 \mathrm{kHz}$ in magnitude and are well within the theory uncertainties (CODATA 2018 uncertainties are not relevant here). The most stringent theory-experiment agreement is found for $\Delta f_{21,19}$, within the roughly $0.7-\mathrm{kHz}$ theory uncertainty, but ten times less stringent than the experimental uncertainty would allow.

In view of the relatively large uncertainties for $f_{\text {spin }, i}^{\text {(theor }}$ above, we introduce a novel way of comparing experiment with theory, using composite frequencies defined as $f_{\mathrm{c}}=\sum_{i} b_{i} f_{i}$, with appropriate weights $b_{\mathrm{i}}$. We aim to find composite frequencies with small theory uncertainty, and therefore must suppress the contribution of the spin energies' uncertainties without suppressing

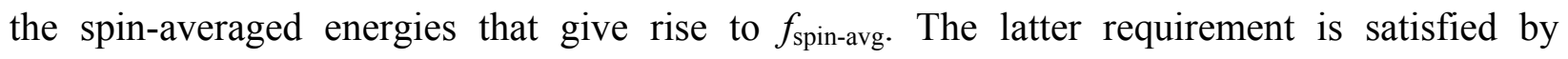
imposing the 'normalization' condition $\sum_{i} b_{i}=1$, so that $f_{\mathrm{c}}=f_{\text {spin-avg }}+f_{\text {spin, }}$, with $f_{\text {spin, }}=\sum_{i} b_{i} f_{\text {spin }, i}$. The former requirement is implemented by finding the composite frequency that minimizes the theory uncertainty. We use a conservative measure of theory uncertainty that does not assume any relationship between the theory errors of $\left(E_{4}, E_{4}^{\prime},\right)$ and of $\left(E_{5}, E_{5}^{\prime},\right)$ : $\bar{u}\left(f_{\text {spin }, c}^{\text {(theor) }}\right)=\sum_{k}\left(\left|\sum_{i} b_{i} \gamma_{i, k}^{\prime} E_{k}^{\prime}\right|+\left|\sum_{i} b_{i} \gamma_{i, k} E_{k}\right|\right) \varepsilon_{\mathrm{k}}$. The solution $\left\{b_{i}\right\}$ is found numerically (see 'Composite frequencies' in Methods), $f_{\text {spin,c }}^{\text {(theor }}\left(\left\{b_{i}\right\}\right)=934.635 \mathrm{kHz}$, with negligible uncertainty $\bar{u}\left(f_{\text {spin,c }}^{\text {(ther) }}\right)=0.001 \mathrm{kHz}$. We note that this approach for eliminating the spin-energy-related uncertainty is complementary to the more general method recently proposed by some of us in ref. ${ }^{36}$, where the composite frequency is equal to $f_{\text {spin-avg. }}$.

From the experimental composite frequency, we deduce the experimental spin-averaged frequency 


$$
\begin{aligned}
f_{\text {spin-arg }}^{(\exp )} & =f_{\mathrm{c}}^{(\exp )}\left(\left\{b_{i}\right\}\right)-f_{\text {spin,c }}^{(\text {theor })}\left(\left\{b_{i}\right\}\right) \\
& =1,314,925,752.910(17)_{\exp } \mathrm{kHz}\left(u_{\mathrm{r}}=1.3 \times 10^{-11}\right)
\end{aligned}
$$

The theory uncertainty (via $f_{\text {spin,c }}^{\text {(ther) }}$ ) is negligible and is therefore not indicated.

\section{QED test and determination of fundamental constants}

A comparison of equations (1) and (2) indicates that our experiment and theory achieve a successful test of three-body physics with a combined fractional uncertainty of $4.8 \times 10^{-11}$ $(0.064 \mathrm{kHz})$, limited by CODATA 2018 uncertainties. Comparing the total uncertainty of $f_{\text {spin-avg }}^{\text {(theor }}$ with the QED contributions listed in Table 2, we see that it is close to the QED contribution of highest calculated relative order of $\alpha^{6}, f^{(6)} \approx 0.070(18) \mathrm{kHz}$. Therefore, more specifically, our experiment furnishes a test of QED at the relative order of $\alpha^{6}$. According to theory, the contributions to $f^{(2)}$ stemming from the finite proton root-mean-square charge radius $r_{\mathrm{p}}$ and the deuteron charge radius $r_{\mathrm{d}}$ with their CODATA2018 uncertainties are $-0.644(3) \mathrm{kHz}$ and $-4.120(3) \mathrm{kHz}$, respectively. The sum of these contributions is put in evidence by our experiment-theory comparison, with a fractional uncertainty of $1.4 \%$.

Our experiment-theory agreement is obtained when including in the hyperfine structure calculation the contribution of the deuteron quadrupole moment $Q_{d}$, quantified by the coefficient $E_{9}^{\prime} \propto Q_{d}$. This contribution is observed here in an MHI for the first time. From the measured hyperfine structure we can extract, independently of any QED contributions, a value for $Q_{\mathrm{d}}$ with $1.5 \%$ fractional uncertainty (Methods).

The experiment-theory agreement can also be used to set improved limits to the hypothetical existence of a spin-averaged fifth force between a proton and a deuteron (Fig. 3, Methods). Compared with previous bounds from MHI spectroscopy, the improvement is a factor of 21 or more for force ranges $\lambda>1 \AA$.

We can obtain the combination $R_{\infty} m_{\mathrm{e}} / \mu_{\mathrm{pd}}$ of fundamental constants from any of the measured rotational frequencies $f_{i}^{(\exp )}$ and the corresponding ab initio value $f_{i}^{(\text {theor })}$. However, the highest precision is obtained by instead choosing the composite frequency $f_{\mathrm{c}}$ or the spinaveraged frequency, because their spin-structure theory uncertainty is suppressed to a negligible level. Furthermore, we note that the ab initio calculation is performed assuming trial values for 
$m_{\mathrm{e}} / m_{\mathrm{p}}$ and $m_{\mathrm{e}} / m_{\mathrm{d}}$, and naturally yields the rotational frequencies in atomic units (independent of Rydberg constant value), $f_{i}^{(\text {theor }, n)} \approx 1.998 \ldots \times 10^{-4}$ a.u.. From these, we compute the scaled, dimensionless values $F_{i}^{(\text {theor })}=\left(\mu_{\mathrm{pd}} / m_{\mathrm{e}}\right) f_{i}^{(\text {theor }, n)} / 1$ a.u.. These have an important dependence on $r_{\mathrm{p}}$ and $r_{\mathrm{d}}$. The dependence on other fundamental constants is weak, compared to their uncertainties, the largest being $\partial \ln F_{i}^{(\text {theor })} / \partial \ln \left(m_{\mathrm{e}} / \mu_{\mathrm{pd}}\right) \approx 4 \times 10^{-3}$. Because of this smallness, it is consistent to use the CODATA 2018 values of the fundamental constants in the computation of $F_{i}^{(\text {theor })}$. This results in

$$
R_{\infty} m_{\mathrm{e}}\left(m_{\mathrm{p}}^{-1}+m_{\mathrm{d}}^{-1}\right)=\frac{f_{\text {spin-avg }}^{(\text {(exp) }}}{2 c F_{\text {spin-avg }}^{(\text {theor) }}}=8,966.20515050(12)_{\text {exp }}(12)_{\text {theor }}(4)_{\text {CODATA2018 }} \mathrm{m}^{-1}\left(u_{\mathrm{r}}=2.0 \times 10^{-11}\right)
$$

where the third uncertainty is due to the proton and deuteron radius uncertainties. The value is in agreement with the CODATA 2018 value of 8,966.205 $15041(41) \mathrm{m}^{-1}\left(u_{\mathrm{r}}=4.6 \times 10^{-11}\right)$ (Fig. 4). It results from atomic hydrogen spectroscopy (providing $R_{\infty}$ ), hydrogen-like ion spin resonance spectroscopy $\left(m_{\mathrm{e}}\right)$ and Penning trap mass spectrometry $\left(m_{\mathrm{p}}, m_{\mathrm{d}}\right)$. Our result's total uncertainty is smaller by a factor of 2.4 compared to the CODATA2018 value and ranks among the most precise measurements of a fundamental constant combination.

Owing to the comparatively small CODATA 2018 uncertainty of $R_{\infty}$, our improved uncertainty impacts mostly the mass ratio sum $m_{\mathrm{e}}\left(m_{\mathrm{p}}^{-1}+m_{\mathrm{d}}^{-1}\right)$. Combining equation (3) with the CODATA 2018 values of $R_{\infty}, m_{\mathrm{e}} / \mathrm{u}$ and $m_{\mathrm{d}} / \mathrm{u}$ yields the proton mass

$$
m_{\mathrm{p}} / u=1.007276466605(20)_{\exp }(21)_{\text {theor }}(45)_{\text {CODATA2018 }}
$$

in excellent agreement with the recent most precise direct measurement ${ }^{40}$

$$
m_{\mathrm{p}} / u=1.007276466598(16)_{\text {stat }}(29)_{\text {syst }}
$$

Taking into account a recent Penning trap measurement of $m_{\mathrm{d}} / m_{\mathrm{p}}\left(\right.$ ref. ${ }^{41}$ ), we also obtain the proton-to-electron mass ratio

$$
m_{\mathrm{p}} / m_{\mathrm{e}}=1,836.152673449(24)_{\exp }(25)_{\text {theor }}(13)_{\text {CODATA2018,Fink-Myers }}
$$

$\left(u_{\mathrm{r}}=2.0 \times 10^{-11}\right)$ in excellent agreement but approximately two times more accurate than the most precise value, obtained by combining two published measurements in Penning traps ${ }^{40,42}$ : $m_{\mathrm{p}} / m_{\mathrm{e}}=1,836.152673374(78)_{\text {exp. }}$. 


\section{Conclusion}

The performance of the recently introduced TICTES technique for rotational spectroscopy has been improved by more than two orders in both resolution and accuracy, reaching a fractional FWHM linewidth of $3 \times 10^{-12}$ and a fractional uncertainty of $1.3 \times 10^{-11}$. This vastly higher performance compared with traditional techniques can be of general relevance to the field of precision molecular physics.

Precise measurements of several rotational hyperfine components of $\mathrm{HD}^{+}$and suppression of the impact of the limited accuracy of the ab initio theory of the spin structure allowed us to establish agreement between experiment and theory at the $5 \times 10^{-11}$ level, limited by uncertainties of the CODATA 2018 fundamental constants. To the best of our knowledge, this represents the most accurate test of a molecular physics prediction to date and also provides the most accurate experiment-theory comparison for any three-body quantum system ${ }^{2,43-45}$. Specifically, we confirmed the combination of the QED contributions of $\alpha^{5}$ relative order, of the proton finite size contribution and of the deuteron finite size contribution, with uncertainty equal to $0.7 \%$ of the total contribution. A strongly improved upper bound for a new force between a proton and a deuteron was set.

Spin-energy differences were experimentally determined with three orders smaller uncertainty than previously ${ }^{12}$. The best (effective) line resolution for spin energy is one order higher and the accuracy is 30 times higher than the benchmark experiment on the spin structure of $\mathrm{H}_{2}^{+}$, which has stood unchallenged for 50 years. The spin-energy predictions were confirmed within the uncertainties of the theory predictions, the smallest uncertainty being $0.7 \mathrm{kHz}$. As the experimental uncertainties are much lower, the obtained spin-energy data offer new benchmark values for future improved ab initio theory of the spin structure.

We deduced the combinations $R_{\infty} m_{\mathrm{e}}\left(m_{\mathrm{p}}^{-1}+m_{\mathrm{d}}^{-1}\right)$ and $m_{\mathrm{e}} / m_{\mathrm{p}}$ of fundamental constants with $2.0 \times 10^{-11}$ fractional uncertainty, 2.4 and 3.0 times smaller, respectively, than the CODATA 2018 uncertainties. The proton mass in atomic mass units was determined with the same uncertainty as in CODATA 2018. Interestingly, for the first time,fundamental constants have been determined with competitive uncertainty making use of the rotational motion of a physical system. 
Our result also provides independent evidence of the correctness of some of the most precise measurements in atomic and particle physics: Rydberg constant determination via hydrogen spectroscopy, electron mass determination via the bound-electron $g$-factor, and proton mass and deuteron mass determination via cyclotron motion. Our measurement on a three-body quantum system thus provides an independent link between these one- and two-body systems. The substantial changes introduced in the CODATA 2018 adjustments of the fundamental constants are confirmed. In particular, the predicted $\mathrm{HD}^{+}$transition frequency is shifted by $0.063 \mathrm{kHz}$ when the CODATA 2014 proton root-mean-square charge radius and Rydberg constant are replaced by the values deduced from the muonic hydrogen experiment (as in CODATA 2018). Our experimental frequency is consistent with the prediction based on these most recent values, within the combined experiment-theory uncertainty of $0.025 \mathrm{kHz}$.

Beyond the present results, our work has important implications for the near future. First, we suppose that in the spectroscopy of vibrational transitions a similar absolute systematic uncertainty can be achieved as in rotational spectroscopy, because the systematic shifts will not increase substantially with transition frequency. Indeed, the shifts depend on the size of the coefficients of appropriate hamiltonians, and these coefficients do not vary substantially between the levels. If an optical spectroscopic technique with spectral resolution at the roughly $10-\mathrm{Hz}$ level becomes available, total experimental uncertainties on the $10^{-13}$ to $10^{-14}$ level could come into reach. Second, our composite frequency approach obviates the need for a more precise spinstructure theory, both for rotational and vibrational transitions. Therefore, more precise QED calculations of the spin-averaged rotational and vibrational frequencies are both sufficient and well worth pursuing. If this challenging programme is successful, the precision of fundamental constants derived from $\mathrm{HD}^{+}$spectroscopy will further improve. Specifically, the combination of rotational and vibrational spectroscopy results and ab initio theory will eventually allow the determination of the fundamental constants $R_{\infty}, m_{\mathrm{e}} / \mu_{\mathrm{pd}}, r_{\mathrm{p}}$ and $r_{\mathrm{d}}$ independently rather than in combination, with accuracies competitive with or better than CODATA 2018, and testing QED without limitation by the uncertainties of the fundamental constants. 
1. Karshenboim, S. G. (ed.) Precision Physics of Simple Atoms and Molecules (Lecture Notes in Physics, Vol. 745, Springer-Verlag, 2008).

2. Pachucki, K., Patkóš, V. \& Yerokhin, V. A. Testing fundamental interactions on the helium atom. Phys. Rev. A 95, 062510 (2017). $\underline{\text { CrossRef }}$

3. Leach, C. A. \& Moss, R. E. Spectroscopy and quantum mechanics of the hydrogen molecular cation: a test of molecular quantum mechanics. Annu. Rev. Phys. Chem. 46, 55-82 (1995). CrossRef

4. Roth, B. et al. in Precision Physics of Simple Atoms and Molecules (ed. Karshenboim, S. G.) 205-232 (Lecture Notes in Physics, Vol. 745, Springer-Verlag, 2008).

5. Wing, W. H., Ruff, G. A., Lamb, W. E. \& Spezeski, J. J. Observation of the infrared spectrum of the hydrogen molecular ion $\mathrm{HD}^{+}$. Phys. Rev. Lett. 36, 1488-1491 (1976). CrossRef

6. Arcuni, P. W., Fu, Z. W. \& Lundeen, S. R. Energy difference between the $(v=0, R=1)$ and the $(v=0, R=3)$ states of $\mathrm{H}_{2}{ }^{+}$, measured with interseries microwave spectroscopy of $\mathrm{H}_{2}$ Rydberg states. Phys. Rev. A 42, 6950-6953 (1990). $\underline{\text { CrossRef }}$

7. Carrington, A., McNab, I. R., Montgomerie-Leach, C. A. \& Kennedy, R. A. Vibrationrotation spectroscopy of the $\mathrm{HD}^{+}$ion near the dissociation limit. Mol. Phys. 72, 735-762 (1991). CrossRef

8. Fu, Z. W., Hessels, E. A. \& Lundeen, S. R. Determination of the hyperfine structure of $\mathrm{H}_{2}{ }^{+}(v=0, R=1)$ by microwave spectroscopy of high- $L, n=27$ Rydberg states of $\mathrm{H}_{2}$. Phys. Rev. A 46, R5313-R5316 (1992). CrossRef

9. Critchley, A. D. J., Hughes, A. N. \& McNab, I. R. Direct measurement of a pure rotation transition in $\mathrm{H}_{2}^{+}$. Phys. Rev. Lett. 86, 1725-1728 (2001). $\underline{\text { CrossRef }}$

10. Osterwalder, A., Wüest, A., Merkt, F. \& Jungen, C. High-resolution millimeter wave spectroscopy and multichannel quantum defect theory of the hyperfine structure in high Rydberg states of molecular hydrogen $\mathrm{H}_{2}^{+}$. J. Chem. Phys. 121, 11810-11838 (2004). CrossRef 
11. Koelemeij, J. C. J., Roth, B., Wicht, A., Ernsting, I. \& Schiller, S. Vibrational spectroscopy of $\mathrm{HD}^{+}$with 2-ppb accuracy. Phys. Rev. Lett. 98, 173002 (2007). CrossRef

12. Bressel, U. et al. Manipulation of individual hyperfine states in cold trapped molecular ions and application to $\mathrm{HD}^{+}$frequency metrology. Phys. Rev. Lett. 108, 183003 (2012). CrossRef

13. Haase, C., Beyer, M., Jungen, C. \& Merkt, F. The fundamental rotational interval of para- $\mathrm{H}_{2}{ }^{+}$by MQDT-assisted Rydberg spectroscopy of $\mathrm{H}_{2}$. J. Chem. Phys. 142, 064310 (2015). $\underline{\text { CrossRef }}$

14. Biesheuvel, J. et al. Probing QED and fundamental constants through laser spectroscopy of vibrational transitions in $\mathrm{HD}^{+}$. Nat. Commun. 7, 10385 (2016). $\underline{\text { CrossRef }}$

15. Korobov, V. I., Hilico, L. \& Karr, J.-P. Fundamental transitions and ionization energies of the hydrogen molecular ions with few ppt uncertainty. Phys. Rev. Lett. 118, 233001 (2017). $\underline{\text { CrossRef }}$

16. Alighanbari, S., Hansen, M., Korobov, V. \& Schiller, S. Rotational spectroscopy of cold and trapped molecular ions in the Lamb-Dicke regime. Nat. Phys. 14, 555-559 (2018). CrossRef

17. Jefferts, K. B. Hyperfine structure in the molecular ion $\mathrm{H}_{2}^{+}$. Phys. Rev. Lett. 23, 14761478 (1969). $\underline{\text { CrossRef }}$

18. Schiller, S. \& Korobov, V. Test of time-dependence of the electron and nuclear masses with ultracold molecules. Phys. Rev. A 71, 032505 (2005). $\underline{\text { CrossRef }}$

19. Bakalov, D. \& Schiller, S. The electric quadrupole moment of molecular hydrogen ions and their potential for a molecular ion clock. Appl. Phys. B 114, 213-230 (2014); erratum 116, 777-778 (2014). $\underline{\text { CrossRef }}$

20. Karr, J.-Ph. $\mathrm{H}_{2}{ }^{+}$and $\mathrm{HD}^{+}$: candidates for a molecular clock. J. Mol. Spectrosc. 300, $37-$ 43 (2014). $\underline{\text { CrossRef }}$

21. Schiller, S., Bakalov, D. \& Korobov, V. I. Simplest molecules as candidates for precise optical clocks. Phys. Rev. Lett. 113, 023004 (2014). CrossRef 
22. Beyer, A. et al. The Rydberg constant and proton size from atomic hydrogen. Science 358, 79-85 (2017). $\underline{\text { CrossRef }}$

23. Fleurbaey, H. et al. New measurement of the $1 S-3 S$ transition frequency of hydrogen: contribution to the proton charge radius puzzle. Phys. Rev. Lett. 120, 183001 (2018). CrossRef

24. Bezginov, N. et al. A measurement of the atomic hydrogen Lamb shift and the proton charge radius. Science 365, 1007-1012 (2019). $\underline{\text { CrossRef }}$

25. Antognini, A. et al. Proton structure from the measurement of $2 S-2 P$ Transition Frequencies of Muonic Hydrogen. Science 339, 417-420 (2013). CrossRef

26. Grémaud, B., Delande, D. \& Billy, N. Highly accurate calculation of the energy levels of the molecular ion. J. Phys. B 31, 383 (1998). CrossRef

27. Moss, R. E. Energies of low-lying vibration-rotation levels of $\mathrm{H}_{2}{ }^{+}$and its isotopomers. $J$. Phys. B 32, L89-L91 (1999). CrossRef

28. Taylor, J. M., Yan, Z.-C., Dalgarno, A. \& Babb, J. F. Variational calculations on the hydrogen molecular ion. Mol. Phys. 97, 25-33 (1999). CrossRef

29. Tiesinga, E., Mohr, P. J., Newell, D. B. \& Taylor, B. N. Values of fundamental physical constants. NIST https://physics.nist.gov/cuu/Constants/index.html (2019).

30. Wolf, F. et al. Non-destructive state detection for quantum logic spectroscopy of molecular ions. Nature 530, 457-460 (2016). $\underline{\text { CrossRef }}$

31. Chou, C. et al. Preparation and coherent manipulation of pure quantum states of a single molecular ion. Nature 545, 203-207 (2017). CrossRef

32. Schneider, T., Roth, B., Duncker, H., Ernsting, I. \& Schiller, S. All-optical preparation of molecular ions in the rovibrational ground state. Nat. Phys. 6, 275-278 (2010).

\section{CrossRef}

33. Roth, B., Blythe, P., Wenz, H., Daerr, H. \& Schiller, S. Ion-neutral chemical reactions between ultracold localized ions and neutral molecules with single-particle resolution. Phys. Rev. A 73, 042712 (2006). $\underline{\text { CrossRef }}$ 
34. Schiller, S., Roth, B., Lewen, F., Ricken, O. \& Wiedner, M. Ultra-narrow-linewidth continuous-wave THz sources based on multiplier chains. Appl. Phys. B 95, 55-61 (2009). CrossRef

35. Bakalov, D., Korobov, V. I. \& Schiller, S. High-precision calculation of the hyperfine structure of the $\mathrm{HD}^{+}$ion. Phys. Rev. Lett. 97, 243001 (2006). CrossRef

36. Schiller, S. \& Korobov, V. I. Canceling spin-dependent contributions and systematic shifts in precision spectroscopy of molecular hydrogen ions. Phys. Rev. A 98, 022511 (2018). CrossRef

37. Bakalov, D., Korobov, V. I. \& Schiller, S. Magnetic field effects in the transitions of the $\mathrm{HD}^{+}$molecular ion and precision spectroscopy. J. Phys. B 44, 025003 (2011) CrossRef; corrigendum 45, 049501 (2012). $\underline{\text { CrossRef }}$

38. Korobov, V. I., Koelemeij, J. C. J., Hilico, L. \& Karr, J.-P. Theoretical hyperfine structure of the molecular hydrogen ion at the 1 ppm level. Phys. Rev. Lett. 116, 053003 (2016). CrossRef

39. Menasian, S. C. \& Dehmelt, H. G. High-resolution study of $(1,1 / 2,1 / 2)-(1,1 / 2,3 / 2)$ HFS transition in $\mathrm{H}_{2}{ }^{+}$. Bull. Am. Phys. Soc. 18, 408 (1973).

40. Heiße, F. et al. High-precision mass spectrometer for light ions. Phys. Rev. A 100, 022518 (2019). $\underline{\text { CrossRef }}$

41. Fink, D. J. \& Myers, E. G. Deuteron-to-proton mass ratio from the cyclotron frequency ratio of $\mathrm{H}_{2}{ }^{+}$to $\mathrm{D}^{+}$with $\mathrm{H}_{2}{ }^{+}$in a resolved vibrational state. Phys. Rev. Lett. 124, 013001 (2020). CrossRef

42. Sturm, S. et al. High-precision measurement of the atomic mass of the electron. Nature 506, 467-470 (2014). CrossRef

43. Pastor, P. C. et al. Absolute frequency measurements of the $2^{3} S_{1} \rightarrow 2^{3} P_{0,1,2}$ atomic helium transitions around 1083 nm. Phys. Rev. Lett. 92, 023001 (2004). CrossRef

44. Hori, M. et al. Buffer-gas cooling of antiprotonic helium to 1.5 to $1.7 \mathrm{~K}$, and antiprotonto-electron mass ratio. Science 354, 610-614 (2016). CrossRef 
45. Rengelink, R. J. et al. Precision spectroscopy of helium in a magic wavelength optical dipole trap. Nat. Phys. 14, 1132-1137 (2018). $\underline{\text { CrossRef }}$

46. Hori, M. et al. Two-photon laser spectroscopy of antiprotonic helium and the antiprotonto-electron mass ratio. Nature 475, 484-488 (2011). CrossRef

47. Udem, T. Quantum electrodynamics and the proton size. Nat. Phys. 14, 632-632 (2018); correction 14, 767 (2018). $\underline{\text { CrossRef }}$ 


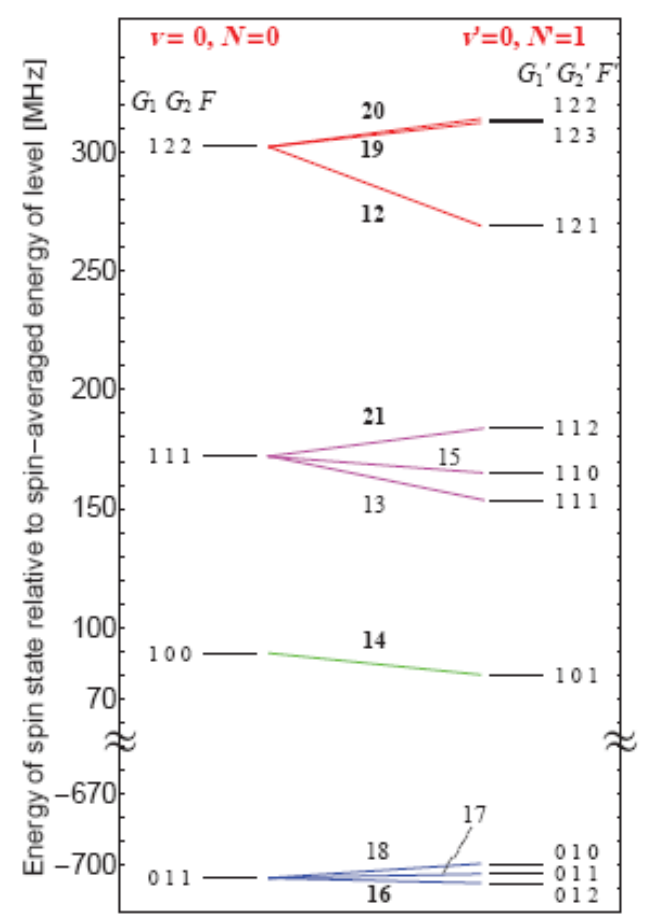

\section{Fig. 1 | Energy diagram of the spin structures and favoured transitions.}

The left side shows the rovibrational ground level $(v=0, N=0)$ and the right side shows the rotationally excited level $\left(v^{\prime}=0, N^{\prime}=1\right)$. The magnetic field is zero. The spin states are labelled by the (in part approximate) quantum numbers $\left(G_{1}, G_{2}\right.$ and $\left.F\right)$. The spin energies $E_{\text {spin }}\left(v, N, G_{1}\right.$, $\left.G_{2}, F\right)$ and $E_{\text {spin }}\left(v^{\prime}, N^{\prime}, G_{1}^{\prime}, G_{2}^{\prime}, F^{\prime}\right)$ are shown as thin black lines. Transitions ('hyperfine components') are numbered according to increasing value of $h f_{\text {spin }, i}=E_{\text {spin }}\left(v^{\prime}, N^{\prime}, G_{1}^{\prime}, G_{2}^{\prime}, F^{\prime}\right)-E_{\text {spin }}\left(v, N, G_{1}, G_{2}, F\right)$, including both favoured and weak transitions. The favoured electric-dipole transitions obey the selection rules $\Delta G_{1}=0, \Delta G_{2}=0$ and $\Delta F=0, \pm 1$. The ten favoured transitions are shown by coloured lines. The rotational transition frequency of a particular hyperfine component is $f_{i}=f_{\text {spin-avg }}+f_{\text {spin, } i \text {, with }} f_{\text {spin-avg }}$ $\approx 1.314 \mathrm{THz}$ and, for favoured transitions, $f_{\mathrm{spin}, i} \approx \mathrm{O}(10 \mathrm{MHz})$. The six components measured in this work are shown by bold numbers in the diagram. 

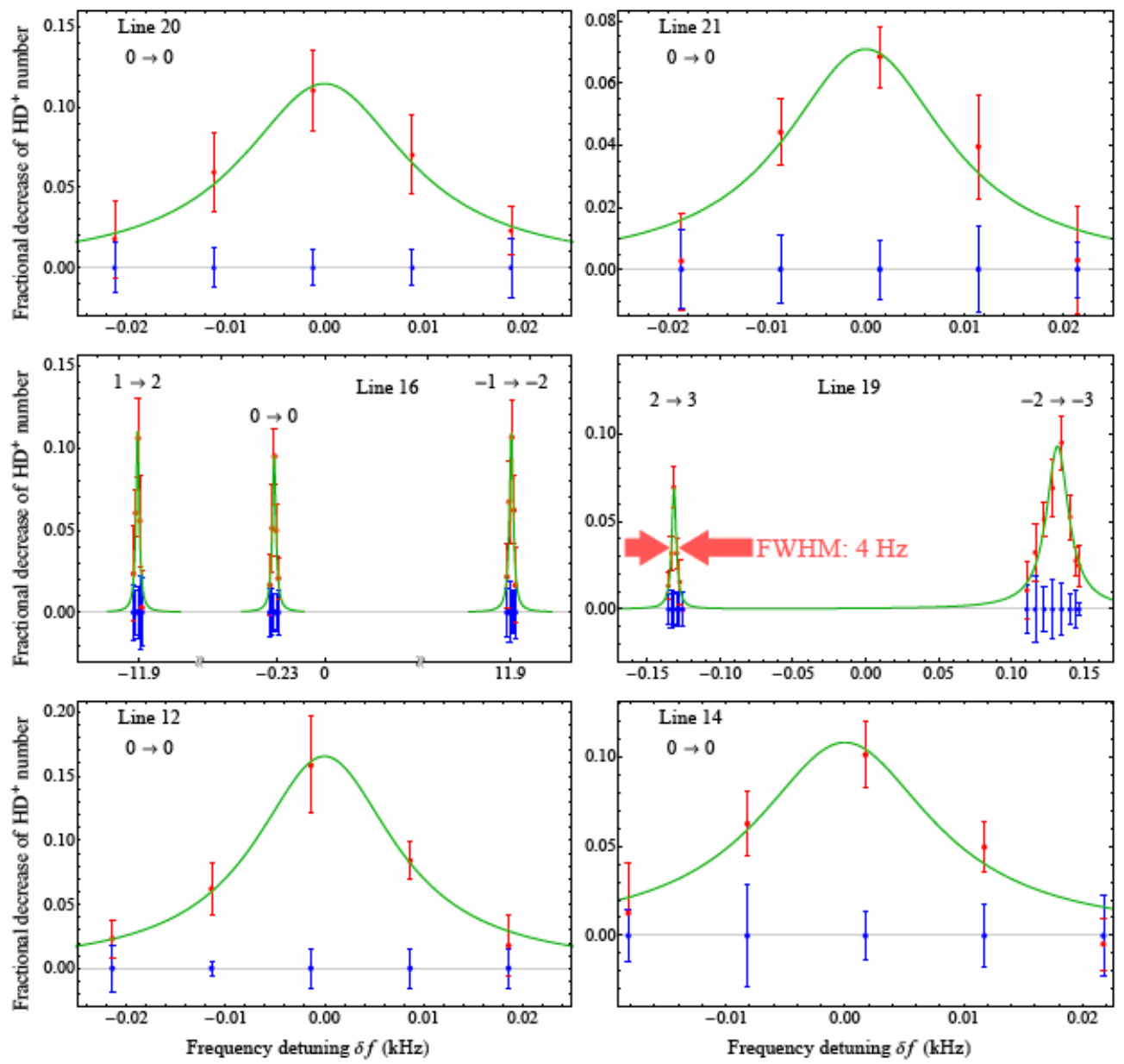

Fig. 2 | Hyperfine components of the fundamental rotational transition of $\mathrm{HD}^{+}$at $1.3 \mathrm{THz}$.

The red and blue points indicate the cases of terahertz radiation on and off (background), respectively. Green lines are Lorentzian fits. The Zeeman components are indicated by the expression $m_{\mathrm{F}} \rightarrow m_{\mathrm{F}}^{\prime}$. The terahertz wave intensity varied and was less than $10 \mathrm{nW} \mathrm{mm} \mathrm{m}^{-2}$. The zero of the frequency scales are set to coincide with the fitted line maxima or means. At each frequency setting, the red and blue data points are both shown with an offset equal to the value of the blue point. Each error bar represents the standard deviation of the mean. The nominal magnetic field is $B_{\text {nom }} \approx 3.0 \times 10^{-5} \mathrm{~T}$ and the trap RF amplitude is approximately $190 \mathrm{~V}$. 


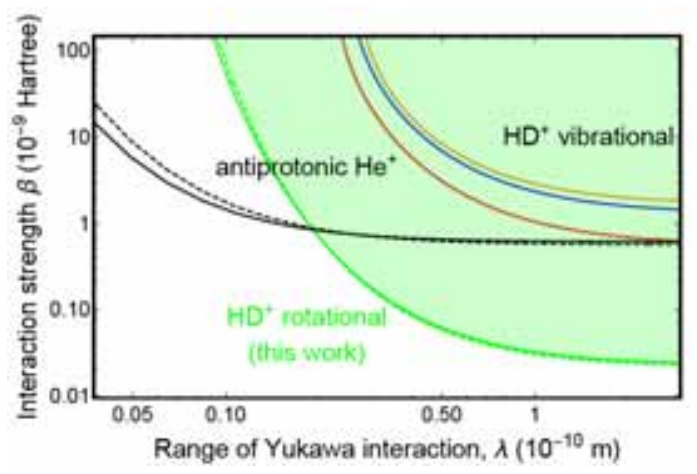

Fig. 3 | Exclusion plot (95\% confidence limit) for a Yukawa-type interaction between a proton and a deuteron, deduced from spectroscopy of MHIs.

The parameter space above the lines is excluded. The assumed interaction is $V_{5}(R)=\beta N_{1} N_{2} \exp (-R / \lambda) / R$, where $R$ is the proton-deuteron distance, $\lambda$ is the interaction range, $N_{1}=1$ and $N_{2}=2$ are the nuclear mass numbers, and $\beta$ is the interaction strength. Green lines, this work (full green, numerical; dashed green, analytical, equation (4) in Methods); red line, ref. ${ }^{14}$; blue line, ref. ${ }^{11}$; orange line, ref. ${ }^{12}$. For comparison, the black lines show the limits for the interaction between the antiproton and the helium-4 nucleus, obtained from two different transitions ${ }^{46}$. See Methods for details. 


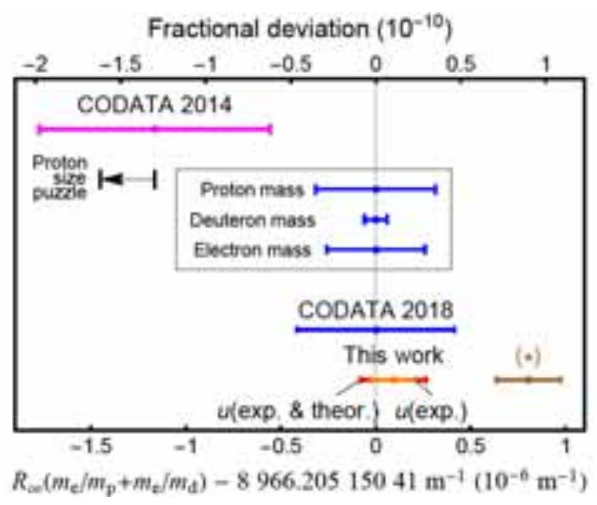

Fig. 4 | Comparison of results of this work with literature values.

In the inner box, we plot the error bars for the CODATA $2018 R_{\infty}\left(m_{\mathrm{e}} / m_{\mathrm{p}}+m_{\mathrm{e}} / m_{\mathrm{d}}\right)$ for the hypothetical cases that the uncertainties of all contributing constants were zero, except for the named constant. The black arrow indicates the shift of the CODATA 2014 value for a change $\Delta R_{\infty}=-0.00035 \mathrm{~m}^{-1}$ corresponding to the 'proton size puzzle' ${ }^{, 4}$. The brown data point (*) shows the result of the present work when the CODATA 2014 values of $r_{\mathrm{p}}$ and $r_{\mathrm{d}}$ are used in $f_{\text {spin-avg }}^{\text {(theor) }}$, instead of the CODATA 2018 values resulting from muonic hydrogen spectroscopy. 
Table 1 | Experimental rotational frequencies, and comparison with theoretical ab initio frequencies

\begin{tabular}{cccccccc}
\hline Line $i G_{1} G_{2} F \rightarrow G_{1} G_{2} F$ & $f_{i}^{(\exp )}$ & $u\left(f_{i}^{(\text {exp })}\right)$ & $f_{i}^{(\text {theor })}$ & $u\left(f_{\text {spin }, i}^{\text {(theor) }}\right) u\left(f_{\text {spin-avg }}^{\text {(theor) }}\right) u_{\text {CODATA }}\left(f_{i}^{\text {(theor) })}\right.$ \\
\hline 12 & $122 \rightarrow 121$ & 1314892544.276 & 0.040 & 1314892544.23 & 1.2 & 0.018 & 0.061 \\
14 & $100 \rightarrow 101$ & 1314916678.487 & 0.064 & 1314916678.74 & 1.3 & 0.018 & 0.061 \\
16 & $011 \rightarrow 012$ & 1314923618.028 & 0.017 & 1314923617.94 & 0.20 & 0.018 & 0.061 \\
19 & $122 \rightarrow 123$ & 1314935827.695 & 0.037 & 1314935827.58 & 1.2 & 0.018 & 0.061 \\
20 & $122 \rightarrow 122$ & 1314937488.614 & 0.060 & 1314937488.80 & 1.4 & 0.018 & 0.061 \\
21 & $111 \rightarrow 112$ & 1314937540.762 & 0.046 & 1314937540.61 & 0.73 & 0.018 & 0.061
\end{tabular}

Uncertainties are denoted by $u$. Frequency values are in $\mathrm{kHz}$. The theoretical values $f_{i}^{\text {(theor) }}$ were computed using CODATA 2018 constants. The last three columns show the three contributions to the total uncertainty of $f_{i}^{\text {(theor) }}$. Line 16 offers the most stringent comparison, due to its comparatively small theory uncertainty. 
Table 2 | Contributions to the ab initio spin-averaged rotational frequency $f_{\text {spin-avg }}^{(\text {theor }}$

\begin{tabular}{|c|c|c|c|}
\hline$\underset{f^{(0)}}{\text { Term }}$ & $\begin{array}{c}\text { Relative order } \\
1\end{array}$ & $\begin{array}{c}\text { Contribution (kHz) } \\
1,314,886,776.526\end{array}$ & $\begin{array}{c}\text { Origin } \\
\text { Solution of three-body Schrödinger equation }\end{array}$ \\
\hline$f^{(2)}$ & $\alpha^{2}$ & $48,416.268$ & $\begin{array}{l}\text { Relativistic corrections in Breit-Pauli } \\
\quad \text { approximation; nuclear radii }\end{array}$ \\
\hline$f^{(3)}$ & $\alpha^{3}$ & $-9,378.119$ & $\begin{array}{l}\text { Leading-order radiative corrections (for example, } \\
\text { leading-order Lamb shift, anomalous magnetic } \\
\text { moment) }\end{array}$ \\
\hline$f^{(4)}$ & $\alpha^{4}$ & $-65.631(2)$ & $\begin{array}{l}\text { One-loop, two-loop radiative corrections; relativistic } \\
\text { corrections }\end{array}$ \\
\hline$f^{(5)}$ & $\alpha^{5}$ & $3.923(3)$ & $\begin{array}{l}\text { Radiative corrections up to three-loop diagrams; } \\
\text { Wichman-Kroll contribution }\end{array}$ \\
\hline$f^{(6)}$ & $\alpha^{6}$ & $-0.070(18)$ & Higher-order radiative corrections \\
\hline & & $1,314,925,752.896(18)$ & \\
\hline
\end{tabular}

The values were calculated using CODATA 2018 values of the fundamental constants. The main contribution $f^{(0)}$ is of order $c R_{\infty}\left(m_{\mathrm{e}} / \mu_{\mathrm{pd}}\right)$. Recoil corrections (due to finite masses of nuclei) are included fully at the order $\alpha^{2}$; the leading recoil corrections proportional to $m_{\mathrm{e}} / m_{\mathrm{p}}$ or $m_{\mathrm{e}} / m_{\mathrm{d}}$ are included at the order $\alpha^{3}$. Contributions due to the finite size of the nuclei are included in the $f^{(2)}$ term ${ }^{15}$. The one-loop contribution from $\mu^{+}-\mu^{-}$vacuum polarization is included in $f^{(3)}$. The estimated fractional theory uncertainty of the spin-averaged frequency is $u_{\mathrm{r}}=1.4 \times 10^{-11}$ $\left(u\left(f_{\text {spin-avg }}^{\text {(ther })}\right)=0.018 \mathrm{kHz}\right)$. The impact of the fundamental constants' uncertainties is given in the text. The change in the value of $f^{(0)}$ from CODATA 2014 to CODATA 2018 has contributions of $-0.041 \mathrm{kHz}$ from the Rydberg constant adjustment and $0.213 \mathrm{kHz}$ from the particle masses adjustments. The change in the value of $f^{(2)}$ due to the proton and deuteron charge radii adjustments is $0.104 \mathrm{kHz}$. 


\section{METHODS}

\section{Experimental procedure}

We simultaneously trapped $\mathrm{Be}^{+}$and $\mathrm{HD}^{+}$ions in a linear RF trap driven at $14.16 \mathrm{MHz}$ (Extended Data Fig. 1). The distance between the trap centre and the RF electrodes was $4.3 \mathrm{~mm}$. For translational cooling of the molecular ions, we laser-cooled the atomic ions with a laser at $313 \mathrm{~nm}$ and the $\mathrm{HD}^{+}$ions were sympathetically cooled via electrostatic interactions with the $\mathrm{Be}^{+}$ ions. We estimated the ion secular temperature as about $30 \mathrm{mK}$. Typically, roughly $10^{2} \mathrm{HD}^{+}$ions were trapped together with about $2 \times 10^{3} \mathrm{Be}^{+}$ions. The number of trapped $\mathrm{HD}^{+}$ions affects the spectral resolution of the rotational transitions, since the Lamb-Dicke regime can only be reached when the ions' displacements in the transverse direction are much smaller than the transition wavelength.

Black-body radiation populates the excited rotational levels of the ground vibrational state until a thermal equilibrium population is reached. We counteracted this by pumping the $\mathrm{HD}^{+}$population into the ground rovibrational state using two lasers. They drive the $(0,2) \rightarrow(1$, $1)$ and $(0,1) \rightarrow(2,0)$ transitions, and the spontaneous decay from the respective excited states eventually transfers a large fraction of the $\mathrm{HD}^{+}$ions in the rovibrational ground state. A quantum cascade laser at $5.48 \mu \mathrm{m}$ excited the former transition, and a distributed feedback laser at $2.7 \mu \mathrm{m}$ excited the latter transition.

After rotational cooling, the terahertz radiation was turned on to drive a transition between specific Zeeman components of a specific hyperfine rotational transition. The terahertz wave intensity was controlled with a half-wave plate, a linear polarizer and via the synthesizer output level. A $1.4-\mu \mathrm{m}$ laser selectively excited molecules from the $(0,1)$ level to the $(4,0)$ level. Molecules in this level were rapidly dissociated by a 266-nm laser.

The spectroscopy scheme relies on the ability to determine the relative decrease of the number of trapped $\mathrm{HD}^{+}$ions. Resonant excitation of the $\mathrm{HD}^{+}$ions' radial secular motion with an auxiliary a.c. electric field couples to the $\mathrm{Be}^{+}$ion ensemble, heating it and causing a change in atomic fluorescence. This fluorescence change is approximately proportional to the number of trapped $\mathrm{HD}^{+}$ions. Applying the secular excitation before and after the REMPD and calculating 
the ratio of average fluorescence levels provides the fractional decrease of the number of $\mathrm{HD}^{+}$ ions. See Extended Data Fig. 2.

As the REMPD process removes $\mathrm{HD}^{+}$ions from the trap, repeated loadings are necessary. With one loading of $\mathrm{Be}^{+}$, approximately 40 loadings of $\mathrm{HD}^{+}$were performed. For each $\mathrm{HD}^{+}$loading, typically five spectroscopy cycles were performed. Each cycle lasted $60 \mathrm{~s}$ and provided one data point.

The magnetic field was $B_{0} \approx 45 \mu \mathrm{T}$, directed along the trap axis, except during rotational spectroscopy/REMPD, when the field was changed to $B \approx 30 \mu \mathrm{T}$ or lower, oriented perpendicular to the trap axis and parallel to the terahertz radiation wave vector (Extended Data Fig. 1). The magnitude and direction of the magnetic field were controlled by three pairs of magnetic coils outside the vacuum chamber.

Owing to the complicated statistics of the ion detection process, we assigned one-half of the FWHM of a line as the statistical uncertainty of a measured transition frequency.

\section{Systematic effects}

As a guide to and comparison with the experimental work, the ab initio values for various systematic effects were taken from our previous calculations. Explicit values for the Zeeman effect are given in ref. ${ }^{37}$. and for the Stark effect in ref. ${ }^{48}$. The ab initio a.c. polarizabilities at the frequency corresponding to the wavelength $266 \mathrm{~nm}$ were computed in ref. ${ }^{16}$.

\section{Trap shift}

Several systematic shifts are expected to give rise to a quadratic dependence on RF amplitude. These include the micromotion-induced Stark shift ${ }^{49}$, phase-offset-induced Stark shift ${ }^{49}$, and a.c. Zeeman shift due to an alternating magnetic field at the trap frequency correlated with the electric trap drive.

We therefore measured the dependence of the six lines (including three Zeeman components for line 16 and two Zeeman components for line 19) on the trap RF amplitude. The typical values chosen for the RF amplitude were $150 \mathrm{~V}, 180 \mathrm{~V}$ and $245 \mathrm{~V}$. The precise RF amplitude value for each measurement was determined by measuring the radial secular frequency of $\mathrm{Be}^{+}$. See Extended Data Fig. 3 for an example of the frequency shift when varying the trap's RF field amplitude. Fits, assuming quadratic dependence, furnish the correction to be 
applied for obtaining each line's extrapolated frequency for zero RF amplitude. The theory of the Stark shift ${ }^{48}$ predicts shifts of the same sign (positive) and of similar value for all components considered here. The experimental data are consistent with this prediction.

\section{Zeeman shift}

Both the linear and quadratic Zeeman shift coefficients vary substantially among Zeeman components and hyperfine components (compare, for example, lines 16 and 19 in Fig. 2). The frequency splitting of the two Zeeman components $16_{ \pm}$together with the theoretical linear Zeeman splitting coefficient $\left(7.98 \mathrm{kHz} \mu \mathrm{T}^{-1}\right.$ (ref. ${ }^{37}$ )) allows the determination of the (time- and ensemble-averaged) magnetic field affecting the molecular ions. For the data shown in Fig. 2, the nominal magnetic field $B_{\text {nom }}=2.98(3) \times 10^{-5} \mathrm{~T}$ is consistent with the value deduced using spectroscopy of the co-trapped beryllium ions ${ }^{50}$. The observed linewidth of the $16_{ \pm}$Zeeman components indicates that the magnetic field is homogeneous to at least 1 part in 30 over the molecule sample.

We measured the frequencies at three different values of magnetic field, for RF amplitudes close to the nominal value of $190 \mathrm{~V}$. Since the RF amplitude varied slightly for the individual measurements, each measured frequency was corrected for the trap shift.

To obtain the $B \rightarrow 0$ extrapolated frequency, $f_{i}^{(\exp )}$, for each line, we fitted to the measured line frequencies $f_{i}^{(\exp )}(B)$ the sum of $f_{i}^{(\exp )}$ plus a quadratic-in- $B$ and/or linear-in- $B$ dependence, depending on the type of Zeeman component. As an accurate measure of the magnetic field, we used the splitting $f_{16_{-}}-f_{16_{+}}$. For $m_{\mathrm{F}}=0 \rightarrow 0$ Zeeman components, we assumed a quadratic-in- $B$ dependence. For the two components $19_{ \pm}$and for the two components $16_{ \pm}$, we allowed for independent linear-in- $B$ shift coefficients $\alpha_{i,+}, \alpha_{i,-}$. For $f_{16_{+}}, f_{16_{-}}$, we added to the fit functions the quadratic Zeeman shift predicted by theory. From the fits, we found that the 'positive' and 'negative' shift coefficients of a given line are close: $\alpha_{19,-,} \approx \alpha_{19,+}$ and $\alpha_{16,-} \approx \alpha_{16,+}$.

The input data for the magnetic-field dependence fit are the trap-field-extrapolated line

frequencies. The reported uncertainty of each $f_{i}^{(\exp )}$ contains both the uncertainty of the magnetic-field extrapolation and the uncertainty due to the trap-field extrapolation. 
The magnetic field is produced by three solenoids. They were characterized with a magnetic probe before closing the vacuum chamber. We find the field value deduced from the solenoids' currents agrees with the value deduced from the splitting $f_{16_{-}}-f_{16_{+}}$, within the experimental uncertainty of the former.

\section{Trap-induced a.c. Zeeman shift}

This effect would show up as a variation of the splitting between two Zeeman components with the trap RF amplitude. The $19_{ \pm}$components were measured at $245 \mathrm{~V}$ and $154 \mathrm{~V}$, at the nominal magnetic field. Their frequency difference did not change, indicating a negligible a.c. Zeeman shift.

\section{Light shift due to cooling laser}

The 313-nm cooling laser permanently irradiates the ion cluster, including when the terahertz wave is on. Its nominal power is $100 \mu \mathrm{W}$ and the beam radius is $0.25 \mathrm{~mm}$. We measured the effect of a change of the 313-nm laser intensity on $f_{16}$. No shift was discernible at the $10-\mathrm{Hz}$ level upon increase of the power by a factor of four.

We computed the scalar, tensor and vector polarizabilities of the rovibrational levels at $\lambda=313 \mathrm{~nm}$ using high-precision variational wavefunctions, similar to ref. ${ }^{48}$, obtaining $\alpha_{\mathrm{s}}(v=0$, $L=1)=3.5054, \alpha_{\mathrm{t}}(v=0, L=1)=-0.955, \alpha_{\mathrm{s}}(v=0, L=0)=3.4961$ and $\alpha_{\mathrm{t}}(v=0, L=0)=0$, in atomic units. The vector polarizabilities are negligible. The computed light shift is of the order of $0.01 \mathrm{~Hz}$. We therefore set the correction due to the 313 -nm wave intensity to zero.

\section{Line pulling}

We have no observational evidence that Zeeman components, or micromotion-induced sidebands of other Zeeman components, could affect the measured transitions. The small linewidths of the measured transitions are important in this respect. We did not observe any change of $f_{16_{+}}, f_{16_{-}}$ and $f_{16_{0}}$ at the $10-\mathrm{Hz}$ level upon a $500-\mathrm{Hz}$ change of the trap frequency. 


\section{d.c. offsets}

For every measurement reported in the manuscript, the $\mathrm{HD}^{+}$ions are located along the symmetry axis of the $\mathrm{Be}^{+}$ion cluster. An offset of $10 \mathrm{~V}$ was applied to an electrode to displace the beryllium crystal by about $100 \mu \mathrm{m}$ from the trap axis along the radial direction. We observed that this offset potential does not have an effect on the position of the $\mathrm{HD}^{+}$ions, as also found in molecular dynamics simulations ${ }^{16}$. We measured the frequency shift of $f_{19}$ caused by this offset potential to be $1(10) \mathrm{Hz}$. Possible day-to-day variations of the trap compensation voltage are a small fraction of the applied offset. Therefore, the size and uncertainty resulting from these variations are negligible.

\section{Light shift due to the two REMPD lasers}

The shift due to the 1.4- $\mu \mathrm{m}$ laser and 266-nm laser waves present during spectroscopy has been determined by performing spectroscopy in a different mode, alternating terahertz irradiation and REMPD laser irradiation. It has been measured for all lines and all Zeeman components discussed here. The shifts are smaller than or equal to $0.039(17) \mathrm{kHz}$ in absolute value. The measured shifts and their uncertainties are used as corrections.

\section{Other shifts}

According to theoretical calculations, the black-body radiation shift ${ }^{48}$ and the molecular electric quadrupole shift ${ }^{51}$ can be neglected at the present level of accuracy.

\section{Data analysis}

Extrapolation of the measured frequencies to zero magnetic field and zero trap amplitude is done by a standard least-squares method. Standard formulae for the propagation of uncertainties are applied.

\section{Spin coefficients, their uncertainties, and sensitivity of the transition frequencies to the spin coefficients}

To allow for an accurate comparison between experiment and ab initio theory, we performed a substantially more accurate computation of the spin-structure coefficients of $\mathrm{HD}^{+}$compared with our earlier work ${ }^{35}$. We extended the approach developed in ref. ${ }^{38}$ and the relevant matrix elements were calculated to ten significant digits. Values of the two spin-structure coefficients 
for the lower level, $E_{4}$ and $E_{5}$, and the nine coefficients for the upper level, $E_{1}^{\prime}, \ldots, E_{9}^{\prime}$ are reported in the Extended Data Table 1. Using these coefficients in the diagonalization of the spin-structure Hamiltonian of ref. ${ }^{35}$, we obtain the spin frequencies $f_{\text {spin, } i}$ (Extended Data Table 1).

The largest spin-structure coefficients, $E_{4}, E_{4}^{\prime}, E_{5}$ and $E_{5}^{\prime}$, have theoretical fractional uncertainties of approximately $\varepsilon_{4} \approx \varepsilon_{5} \approx 1 \times 10^{-6}=\varepsilon_{\mathrm{F}}$. This estimate is confirmed by comparison of the theoretical predictions of the molecule $\mathrm{H}_{2}^{+}$, calculated with the same theoretical approach, with the experimental results of refs. ${ }^{17,39}$. For a given vibrational level, the rotational dependence of the neglected terms in $E_{4}$ and $E_{5}$ is nearly zero, because these are contact terms determined by the electronic wave function, which depends very weakly on $N$. This allows us to assume that the neglected terms in $\left(E_{4}, E_{4}^{\prime}\right)$ and in $\left(E_{5}, E_{5}^{\prime}\right)$ are essentially equal, respectively.

Under this assumption, the theory uncertainty of a spin frequency due to these coefficients $k=4,5$ is set to $u_{k}=\left|\gamma_{i, k}^{\prime} E_{k}^{\prime}-\gamma_{i, k} E_{k}\right| \varepsilon_{\mathrm{F}}$, where $\gamma_{i, k}=-\partial f_{\mathrm{spin}, i} / \partial E_{\mathrm{k}}$ is the derivative of the spin energy of the lower quantum state involved in the transition $i$ with respect to the spin coefficient $E_{\mathrm{k}}$, and $\gamma_{i, k}^{\prime}=-\partial f_{\mathrm{spin}, i} / \partial E_{\mathrm{k}}^{\prime}$ is defined analogously for the upper state. The values of the derivatives are presented in Extended Data Table 1.

The spin Hamiltonian coefficients $E_{4} \approx E_{4}^{\prime}$ and $E_{5} \approx E_{5}^{\prime}$ are similar for the two rotational states, and because the transitions studied here are those between similar spin states, for which $G_{1}=G_{1}^{\prime}, G_{2}=G_{2}^{\prime}$, the spin frequencies are small, $\left|f_{\text {spin, } i}\right| \ll<E_{4}, E_{5}, E_{4}^{\prime}, E_{5}^{\prime}$ and the sensitivities are similar, $\gamma_{i, k}^{\prime} \approx \gamma_{i, k}$. Therefore, we benefit from important reduction of the theory uncertainties $u_{4}$ and $u_{5}$ contributed by these four coefficients. Even in the least favourable case, line 14, the uncertainty contribution is less than or equal to $u_{4}+u_{5} \approx 14 \mathrm{~Hz}\left(1 \times 10^{-11}\right)$, that is, negligible compared with the following contributions.

A second set of coefficients, $E_{1}^{\prime}, E_{6}^{\prime}, E_{7}^{\prime}$, are one to three orders smaller in magnitude, and have estimated fractional uncertainties of $\varepsilon_{1} \approx \varepsilon_{6} \approx \varepsilon_{7} \approx \alpha^{2}=\varepsilon_{0} \approx 5 \times 10^{-5}$. Their absolute uncertainties, $1.5 \mathrm{kHz}$ to $0.06 \mathrm{kHz}$, are at a relevant level. They enter the spin-structure frequency uncertainty with contributions $u_{k}=\left|E_{k}^{\prime}\right| \varepsilon_{0}$. 
The fractional uncertainties of the coefficients $E_{2}^{\prime}, E_{3}^{\prime}, E_{8}^{\prime}, E_{9}^{\prime}$ are similar to $\varepsilon_{0}$, but are not relevant at the present experimental accuracy level because the coefficients themselves are much smaller than the others.

As the details of the theory errors are unknown, the total uncertainty of the spin frequencies is set conservatively as the sum over all $u_{k}$ (instead of the root sum of squares).

The sensitivities $\gamma$ are obtained by first computing the eigenvalues $E_{\mathrm{spin}, i}$ and $E_{\mathrm{spin}, i}^{\prime}$ of the Hamiltonian analytically and then computing analytically their derivatives with respect to the individual coefficients $E_{\mathrm{k}}$ and $E_{\mathrm{k}}^{\prime}$. These derivatives are then evaluated for the set of current theory values for $E_{\mathrm{k}}$ and $E_{\mathrm{k}}^{\prime}$.

\section{Fit of the spin Hamiltonian coefficients}

From the six measured transitions, we can derive information about the spin Hamiltonian coefficients and about the true spin-averaged frequency. Under the previous assumption of equal theory errors for $\left(E_{4}, E_{4}^{\prime}\right)$ and for $\left(E_{5}, E_{5}^{\prime}\right)$, there are six remaining important quantities $\left(E_{1}^{\prime}, E_{4}^{\prime}\right.$, $E_{5}^{\prime}, E_{6}^{\prime}, E_{7}^{\prime}$ and $f_{\text {spin-avg }}$ ), and they can be solved for using a set of equations setting the experimental frequencies equal to the corresponding theoretical frequencies allowing for small deviations from the nominal values. We find $E_{1}^{\prime(\text { fit })}-E_{1}^{\prime(\text { theory })}=0.32(20) \mathrm{kHz}$, having an uncertainty smaller than the theory uncertainty of about $\varepsilon_{\mathrm{F}} E_{1}^{\prime} \approx 1.6 \mathrm{kHz}$. Furthermore, $E_{6}^{\prime(\text { fit })}-E_{6}^{\prime(\text { theory })}=0.5(9) \mathrm{kHz}, E_{7}^{\prime(\text { fit })}-E_{7}^{\prime(\text { theory })}=-0.3(4) \mathrm{kHz} \quad$ and $f_{\text {spin-arg }}^{(\text {fit })}-f_{\text {spin-arg }}^{\text {(theor) }}=-0.05(22) \mathrm{kHz}$.

The shown uncertainties result from the experimental errors and the theory error of $f_{\text {spin-avg }}^{\text {(theor }}$; the theory errors of $E_{2}^{\prime}, E_{3}^{\prime}, E_{8}^{\prime}$ and $E_{9}^{\prime}$ make negligible contributions. The deviations of $E_{4}^{\prime}$ and $E_{5}^{\prime}$ from the nominal values cannot be determined precisely (an aspect that is intrinsic to the favoured transitions), but are consistent with zero.

\section{Composite frequencies}

The coefficients of the composite frequency given in the main text are:

$b_{12}=0.086 \quad 372 \quad 0 \ldots, \quad b_{14}=0.145 \quad 634 \quad 8 \ldots, \quad b_{16}=0.251 \quad 611 \quad 1 \ldots, \quad b_{19}=0.244 \quad 279 \quad 2 \ldots$, $b_{20}=0.1328074 \ldots, b_{21}=0.1392955 \ldots$

We consider alternative composite frequencies. One alternative ansatz for finding a composite frequency is to impose the 'insensitivity conditions' 
$0=\partial f_{c}^{(\text {theor })} / \partial E_{k_{\alpha}}=\sum_{i} b_{i} \gamma_{i, k_{\alpha}}, 0=\partial f_{c}^{(\text {theor })} / \partial E_{k_{\beta}}^{\prime}$ for a suitable subset $\left\{k_{\alpha}, k_{\beta}\right\}$ of spin Hamiltonian coefficients. As discussed above, if we assume correlated errors for the pair $\left(E_{4}, E_{4}^{\prime}\right)$ and $\left(E_{5}\right.$, $E_{5}^{\prime}$ ), then the largest theory uncertainties arise from $E_{1}^{\prime}, E_{6}^{\prime}, E_{7}^{\prime}$. Four experimentally measured transitions are sufficient to satisfy the three insensitivity conditions for these three coefficients. The normalization condition is easily imposed in addition. Considering, for example, the lines $14,16,19$ and 21 , the resulting uncertainty from hyperfine theory is $u\left(f_{\text {spin,c }}^{\text {(theor })}\right) \approx 2 \mathrm{~Hz}$, much smaller than the uncertainty of the spin-averaged frequency $u\left(f_{\text {spin-avg }}^{(\text {theory })} \approx 0.02 \mathrm{kHz}\right.$. Thus, the composite frequency has a substantially reduced theory uncertainty compared with those of the individual hyperfine transitions. $f_{\mathrm{c}}^{\text {(theor) }}$ is then also numerically close to $f_{\text {spin-avg, }}$ $f_{\mathrm{c}}^{\text {(theor) }} \approx f_{\text {spin-avg }}^{\text {(theor }}+2,232 \mathrm{kHz}$. With more available transitions we can impose additional conditions.

A second alternative composite frequency is as follows. As in the main text, we consider a composite frequency that minimizes the spin-coefficients-related uncertainty. If we assume correlated $E$ errors, the linear combination of only three lines, $f_{\mathrm{c}}=b_{14} f_{14}+b_{16} f_{16}+\left(1-b_{14}-\right.$ $\left.b_{16}\right) f_{21}$, yields an uncertainty of $3 \mathrm{~Hz}\left(2.4 \times 10^{-12}\right)$. As in the first alternative, this uncertainty is also much smaller than $u\left(f_{\text {spin-avg }}^{\text {(theory })}\right.$. The coefficients are $b_{14}=0.0814 \ldots, b_{16}=0.615 \ldots$ and $f_{\mathrm{c}}^{\text {(theor) }}=f_{\text {spin-avg }}^{\text {(theor) }}+1524.23 \mathrm{kHz}$. Such optimal solutions exist independently of the concrete values of the estimated theory uncertainties of the $E$ coefficients: if the assumed fractional uncertainties $\varepsilon_{k}$ are doubled, a solution is obtained whose theory uncertainty is correspondingly larger, $6 \mathrm{~Hz}$. The relationship between the solution $f_{\mathrm{c}}^{\text {(theory) }}$ and the cancellation conditions is that the determinant of the sensitivity matrix $\Gamma_{i, k}=\gamma_{i, k}^{\prime}=\partial f_{i}^{(\text {theor })} / \partial E_{k}^{\prime}$, where $i=\{14,16,21\}$ and $k=\{1,6,7\}$, is close to zero (about 0.008). This implies that these three transitions are nearly linearly dependent and allow for a composite frequency that nearly satisfies the cancellation conditions (and the normalization condition).

If the correlation assumption is not made, the optimum composite frequency based on lines 14, 16 and 21 yields a comparatively large spin-energy uncertainty of $0.22 \mathrm{kHz}$. For this reason, in the main text, we determined the composite frequency based on six lines. 
A third example is the composite frequency based on the five lines $14,15,16,19$ and 20 : it yields a theory uncertainty $\bar{u}\left(f_{\text {spini, }}^{(\text {theor })}\right) \approx 3 \mathrm{~Hz}$.

Finally, an example of composite frequency for a vibrational transition is the following. For the transition $(v=0, N=0) \rightarrow\left(v^{\prime}=1, N^{\prime}=1\right)$ the six lines $14,15,16,19,20$ and 21 yield a composite frequency with theory uncertainty $\bar{u}\left(f_{\text {spin, } c}^{\text {(ther) }}\right) \approx 2 \mathrm{~Hz}$. This is only $3 \times 10^{-14}$ relative to the vibrational transition frequency $f_{\text {spin-avg }} \approx 58.6 \mathrm{THz}$.

\section{Fifth force bound}

Given the present results, the $95 \%$ confidence limit to the strength of the fifth force, $\beta_{\max }(\lambda)$, is approximately given by

$$
\begin{aligned}
& N_{1} N_{2}|\Delta Y(\lambda)| \beta_{\text {max }}(\lambda) \approx 2 h u_{\text {tot }}(f), \\
& u_{\text {tot }}(f)^{2}=u\left(f_{\text {spin-avg }}^{(\text {exp })}\right)^{2}+u\left(f_{\text {spin-arg }}^{(\text {theor) }}\right)^{2}+u_{\text {CODATA2018 }}\left(f_{\text {spin-avg }}^{\text {(theor) }}\right)^{2}
\end{aligned}
$$

Here, $\Delta Y(\lambda)$ is obtained numerically from perturbation theory as the difference of the expectation value of $R^{-1} \exp (-R / \lambda)$ in the two rotational states, where $R$ is the internuclear separation in atomic units, and $N_{1}, N_{2}$ were defined previously.

We have also obtained an analytical approximation for the rotational transition case

$$
\beta_{\max }(\lambda) \approx 2 \frac{u_{\text {tot }}\left(f_{\text {rot }}\right)}{f_{\text {rot }}} \frac{e^{R_{e} / \lambda}}{2 N_{1} N_{2}\left(1+R_{e} / \lambda\right)} \frac{R_{e} E_{\text {vib }}^{2}}{E_{\text {rot }}}
$$

where $R_{\mathrm{e}}$ is the equilibrium distance, and $E_{\mathrm{rot}}=f_{\mathrm{rot}} / 2 c R_{\infty}$ and $E_{\mathrm{vib}}$ are the fundamental rotational transition energy and fundamental vibrational transition energy, respectively. They are in atomic units. The previous bounds on $\beta$ are also discussed in ref. ${ }^{52}$.

\section{Electric quadrupole moment of the deuteron}

We are also able to deduce a value for the electric quadrupole moment of the deuteron, $Q_{\mathrm{d}}$. The tensor interaction between $Q_{\mathrm{d}}$ and the electric field gradient within the $\mathrm{HD}^{+}$molecule ${ }^{35}$ contributes to the hyperfine structure. It is quantified by the spin Hamiltonian coefficient $E_{9}^{\prime}=5.666 \mathrm{kHz} \propto Q_{\mathrm{d}}$. The ratio $E_{9}^{\prime} / Q_{\mathrm{d}}$ is available from our theory with small fractional uncertainty $\varepsilon_{0} \approx 5 \times 10^{-5}$. The frequencies of the rotational transition components are sensitive to $E_{9}^{\prime}$ to varying degrees, quantified by $\gamma_{i, 9}^{\prime}$ (see Extended Data). We therefore consider a composite frequency $f_{\mathrm{c}}^{\prime}=\sum_{i} a_{i} f_{i}$ that suppresses the spin-averaged frequency, and thus all QED 
contributions, by imposing $\sum_{i} a_{i}=0$. We determine the weight set $\left\{a_{i}\right\}$ that maximizes the sensitivity-to-uncertainty ratio $\left|\partial f_{\mathrm{c}}^{\prime} / \partial E_{9}^{\prime}\right|^{2} /\left(u\left(f_{\mathrm{c}}^{\prime(\text { theor })}\right)^{2}+u\left(f_{\mathrm{c}}^{\prime(\exp )}\right)^{2}\right)$. We find $a_{12}=-0.216516$ 7, $a_{14}=0.6508068, a_{16}=-0.9098989, a_{19}=-0.9738303, a_{20}=-0.1153690$.

From the comparison of $f_{\mathrm{c}}^{\prime(\text { theor })}$ and $f_{\mathrm{c}}^{\prime(\exp )}$, we then deduce $Q_{\mathrm{d}}^{\prime}=0.282(4) \mathrm{fm}^{2}$. It is consistent with the reference value $Q_{\mathrm{d}}=0.28578(3) \mathrm{fm}^{2}$, obtained from RF spectroscopy of neutral $\mathrm{D}_{2}$ and theory ${ }^{53}$. The precision is expected to improve with progress in MHI spin-structure theory.

48. Schiller, S., Bakalov, D., Bekbaev, A. K. \& Korobov, V. I. Static and dynamic polarizability and the Stark and blackbody-radiation frequency shifts of the molecular hydrogen ions $\mathrm{H}_{2}{ }^{+}, \mathrm{HD}^{+}$, and $\mathrm{D}_{2}^{+}$. Phys. Rev. A 89, 052521 (2014). CrossRef

49. Berkeland, D. J., Miller, J. D., Bergquist, J. C., Itano, W. M. \& Wineland, D. J. Minimization of ion micromotion in Paul trap. J. Appl. Phys. 83, 5025-5033 (1998). CrossRef

50. Shen, J., Borodin, A. \& Schiller, S. A simple method for characterization of the magnetic field in an ion trap using $\mathrm{Be}^{+}$ions. Eur. Phys. J. D 68, 359 (2014). $\underline{\text { CrossRef }}$

51. Bakalov, D. \& Schiller, S. The electric quadrupole moment of molecular hydrogen ions and their potential for a molecular ion clock. Appl. Phys. B 114, 213-230 (2014) CrossRef; corrigendum Appl. Phys. B 116, 777-778 (2014). $\underline{\text { CrossRef }}$

52. Salumbides, E. J., Ubachs, W. \& Korobov, V. I. Bounds on fifth forces at the sub-Å length scale. J. Mol. Spectrosc. 300, 65-69 (2014). CrossRef

53. Pavanello, M., Tung, W.-C. \& Adamowicz, L. Determination of deuteron quadrupole moment from calculations of the electric field gradient in $\mathrm{D}_{2}$ and HD. Phys. Rev. A 81, 042526 (2010). CrossRef 


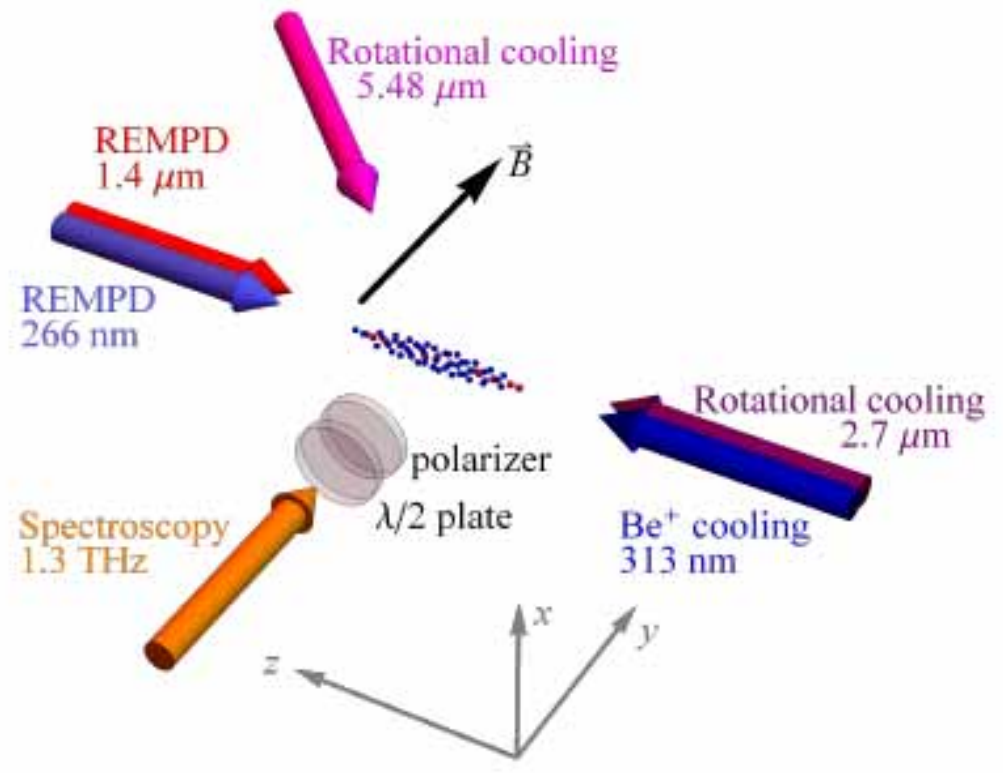

\section{Extended Data Fig. 1 | Conceptual view of the arrangement used for high-resolution spectroscopy of $\mathrm{HD}^{+}$using TICTES.}

The spectroscopy wave $(1.3 \mathrm{THz})$ crosses the ion cluster perpendicular to its long axis, enabling spectroscopy in the Lamb-Dicke regime. The ion cluster comprises atomic $\mathrm{Be}^{+}$ions (blue dots) and $\mathrm{HD}^{+}$molecular ions (red dots). The indicated laser beams implement the Doppler cooling of $\mathrm{Be}^{+}$ions $(313 \mathrm{~nm})$, rotational cooling of $\mathrm{HD}^{+}(2.7 \mu \mathrm{m}$ and $5.48 \mu \mathrm{m})$ and detection by REMPD $(266 \mathrm{~nm}$ and $1.4 \mu \mathrm{m})$. The magnetic field $\boldsymbol{B}$ lifts the degeneracy of Zeeman sublevels during terahertz spectroscopy. The polarizer and the half-wave plate enable adjustment of the polarization and intensity of the terahertz radiation. 


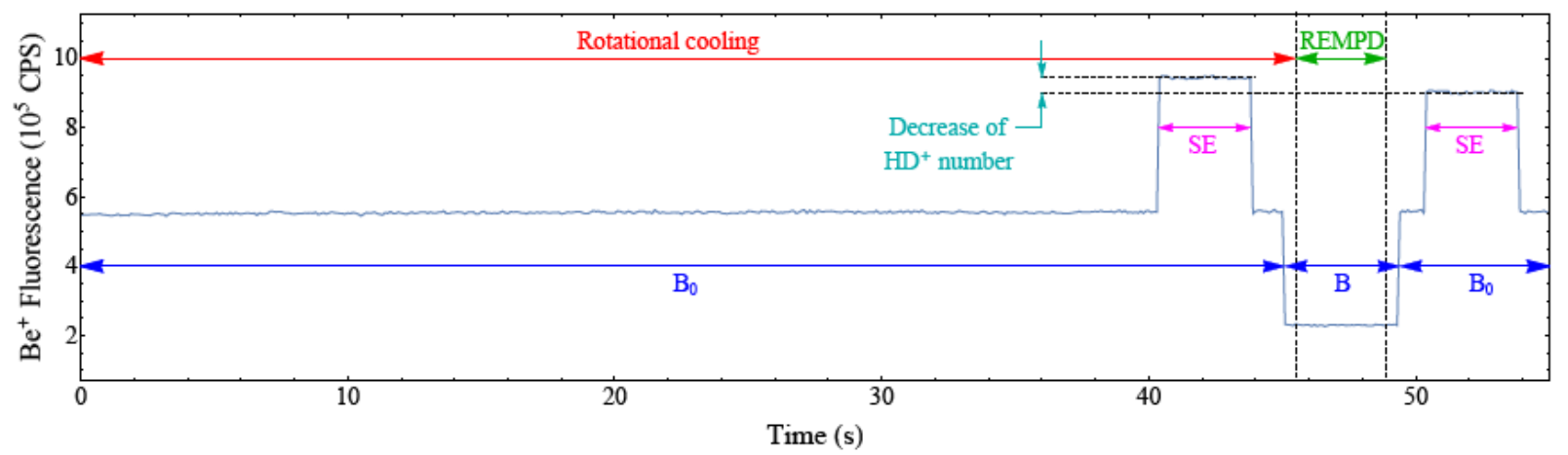

Extended Data Fig. 2 | Beryllium ion fluorescence during one preparation-spectroscopy cycle.

Spectroscopy (terahertz wave on) occurs during the interval marked 'REMPD'. Beryllium laser cooling is on all the time. SE, secular excitation. $\mathrm{B}$, a magnetic flux strength $B$ is applied during REMPD. $\mathrm{B}_{0}$, a strength $B_{0}$ is applied for rotational laser cooling. CPS, counts per second. The signal obtained from the spectroscopy cycle is indicated in cyan. 


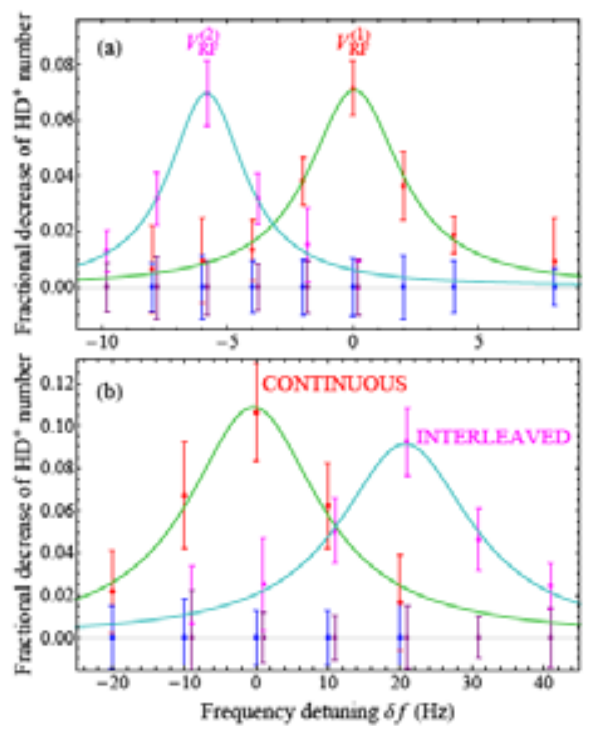

Extended Data Fig. 3 | Systematic shifts of the Zeeman component 19+ of the rotational hyperfine transition line 19.

a, The trap's amplitude is decreased by $2.5 \mathrm{~V}$ from $V_{\mathrm{RF}}^{(1)}$ to $V_{\mathrm{RF}}^{(2)}$. The FWHM linewidth is $4 \mathrm{~Hz}$, corresponding to $3 \times 10^{-12}$ fractional FWHM. b, The light shift induced by the $266 \mathrm{~nm}$ and $1.4 \mu \mathrm{m}$ dissociation lasers, determined by comparing two spectroscopy modes. "Continuous", the lasers are on when the terahertz radiation is applied. "Interleaved", lasers and terahertz radiation are on alternatingly. 


\begin{tabular}{|c|c|c|c|c|c|c|c|c|c|c|c|c|}
\hline & & $\begin{array}{c}\varepsilon_{1}^{\prime} \\
31.98465\end{array}$ & $\begin{array}{c}\varepsilon_{2}^{\prime} \\
-0.03134\end{array}$ & $\begin{array}{c}\mathcal{E}_{3}^{\prime} \\
-0.004810\end{array}$ & $\begin{array}{c}\varepsilon^{\prime} \\
924.56943\end{array}$ & $\begin{array}{c}\mathcal{E}_{5}^{\prime} \\
142.16092\end{array}$ & $\begin{array}{l}\varepsilon_{6^{\prime}} \\
8.61111\end{array}$ & $\begin{array}{l}\mathcal{E}^{\prime} \\
1.32177\end{array}$ & $\begin{array}{c}\mathcal{E}_{8^{\prime}} \\
-0.003057\end{array}$ & $\begin{array}{l}\varepsilon_{9}^{\prime} \\
0.005666\end{array}$ & $\begin{array}{c}\varepsilon_{4} \\
925.39588\end{array}$ & $\begin{array}{c}\varepsilon_{s} \\
142.28781\end{array}$ \\
\hline Line $i$ & $f_{\text {spini }}^{\text {(theck) }}$ & $\gamma_{i, 1}^{\prime}$ & $\gamma_{i, 2}$ & $\gamma_{i, 3}$ & $\gamma_{i, 4}$ & $\gamma_{i, S}$ & $\gamma_{i, 6}^{\prime}$ & $\gamma_{i, 7}$ & $\gamma_{i, 8}^{\prime}$ & $\gamma_{i, 9}$ & $\gamma_{2,4}$ & $\gamma_{i, s}$ \\
\hline 12 & -33.20866 & -0.569 & -0.559 & -1.718 & 0.250 & 0.425 & 0.039 & -3.347 & -3.284 & -2.944 & 0.250 & 0.500 \\
\hline 14 & -9.07415 & -0.429 & -0.386 & 0.654 & 0.249 & -0.908 & -1.028 & 0.834 & 0.721 & -0.502 & 0.250 & -1.000 \\
\hline 16 & -2.13496 & -0.107 & 0.111 & 0.995 & -0.734 & -0.184 & 0.010 & 0.143 & -0.157 & -0.493 & -0.737 & -0.169 \\
\hline 19 & 10.07468 & 0.500 & 0.500 & 1.000 & 0.250 & 0.500 & -0.500 & -1.000 & -1.000 & -0.500 & 0.250 & 0.500 \\
\hline 20 & 11.73591 & -0.225 & -0.265 & -0.510 & 0.250 & 0.500 & 1.734 & 3.439 & 3.559 & 1.764 & 0.250 & 0.500 \\
\hline 21 & 11.78771 & 0.332 & 0.154 & 0.514 & 0.234 & -0.315 & 0.255 & -0.582 & -0.402 & 0.229 & 0.237 & -0.331 \\
\hline
\end{tabular}

\section{Extended Data Table 1 | Spin Hamiltonian coefficients, spin-structure frequencies and} spin-frequency derivatives

$E_{\mathrm{k}}^{\prime}\left(E_{\mathrm{k}}\right)$ are the updated coefficients of the spin Hamiltonian ${ }^{35}$ of the upper (lower) rotational level, in MHz. $f_{\text {spin, }, i}^{\text {(theor }}$ are theoretical spin frequencies in MHz. $\gamma$ are the dimensionless sensitivities of the spin frequencies to the spin Hamiltonian coefficients. $\gamma_{i, k}^{\prime}=\partial f_{\text {spin,i }}^{(\text {theor })} / \partial E_{k}^{\prime}$ refer to the upper state and $\gamma_{i, k}=-\partial f_{\text {spin, }, i}^{(\text {(theor })} / \partial E_{k}$ to the lower state. The entries for line 19 are decimal representations of rational values (see eq. (6) in ref. ${ }^{37}$ ). Note that because of the tracelessness of the spin $\operatorname{Hamiltonian}^{36}, \quad \sum_{i} d_{i} \gamma_{i, k}=0$ and $\sum_{i} d_{i}^{\prime} \gamma_{i, k}^{\prime}=0$, where $d_{i}=(2 F(i)+1) / 36$ and $d_{i}^{\prime}=\left(2 F^{\prime}(i)+1\right) / 36$ are the degeneracies of the respective spin states, and the sum is over the ten favoured transitions $i=12, \ldots, 21$. 